\title{
Formulation of the coupled electrochemical-mechanical boundary-value problem, with applications to transport of multiple charged species
}

\author{
Giovanna Bucci ${ }^{\mathrm{a}, *}$, Yet-Ming Chiang ${ }^{\mathrm{a}}$, W. Craig Carter ${ }^{\mathrm{a}}$ \\ ${ }^{a}$ Massachusetts Institute of Technology, Department of Materials Science and Engineering - \\ 77 Massachusetts Avenue, Cambridge, MA 02139-4307 USA
}

\begin{abstract}
A framework is presented that treats the combined effects of nonlinear elastic deformation, lattice constraints, and electrochemical potentials. The electrochemo-mechanical diffusion potential is derived, and the particular case where ionic species are subject to a crystal lattice constraint is also derived. By combining energy balance and local entropy production, the framework provides a consistent method to treat the evolution of charged species which also carry anelastic deformations in a crystal lattice. The framework is used to derive a finite element formulation that applies to general cases of interest for diffusion of active species in battery electrodes and in fuel cells. We demonstrate the application of the finite element formulation with two cases: ambipolar diffusion and kinetic demixing. The predicted system response demonstrates how mechanical effects cannot be disregarded, even in the presence of dominant electrostatic forces acting on ion transport. A cation rich surface layer is predicted when elastic forces, due to Vegard's stress, participate in the migration of defects in a multicomponent oxides. Simulations show how stress plays a central role in the cation segregation to interfaces, a phenomenon regarded as critical to power and durability of solid oxide fuel cells.
\end{abstract}

Keywords: Electrochemical systems; Nonlinear continuum mechanics; Thermodynamics; Ambipolar Diffusion; Kinetic demixing

\section{Introduction}

Materials for energy conversion and storage have complex microstructures and their physical behavior depends on coupling of multiple mechanisms and driving forces. Macroscopic models that account for such complex phenomena are 5 a materials-modeling challenge. For instance, modeling of electrode and electrolyte materials for Li-ion batteries or solid oxide fuel cells (SOFCs) involves

\footnotetext{
${ }^{*}$ Corresponding author

Email address: bucci@mit.edu (Giovanna Bucci )
}

Preprint submitted to Acta Materialia

November 10, 2015

(C) 2015. This manuscript version is made available under the Elsevier user license http://www.elsevier.com/open-access/userlicense/1.0/ 
diffusion of multiple charged species in a solid medium in the presence of stress, electrostatic, and chemical potential gradients. Inclusion of these multiple fields and transport mechanisms into microstructural models can be performed by 10 finite element methods. However, as a first step in the prediction of timedependent species distribution, induced electric fields, deformation of the solid and the effect of stress on diffusion, it is necessary to construct a rigorous formulation of the coupled physics of mass transport, induced electric fields, and nonlinear continuum mechanics. The purpose is to predict the overall electro-

15 chemical performance-including limiting factors, failure mechanisms, sensitivity to external conditions and material properties-for electro-chemo-mechanical systems with complex microstructures.

The present article proposes a quantitative description of such multifunctional materials drawn from the study of a general non-homogeneous irreversible ther20 modynamic system under the action of multiple physical phenomena. From this analysis, we develop a finite-element formulation and present simulations that demonstrate its applicability to ambipolar diffusion and kinetic demixing. The predictive capability of the model depends on a consistent derivation and on the choice of appropriate numerical procedures to solve it. The formulation 25 provides a foundation for subsequent models which treat the disparate lengthand time-scales in complex battery and SOFC microstructures.

We present a derivation of the driving forces for the time-dependent evolution of the state variables. At each instant in time the system is considered to be in the state of local equilibrium. Mechanical equilibrium will be treated as 30 instantaneous (i.e., static), and the transport of species is transient. To that end, we follow Larché and Cahn (see $\S 7$ in [1]): the most common partial equilibrium occurs when all processes except diffusion have relaxed to equilibrium. The local equilibrium is still guaranteed and "The only suppressed condition is that [the diffusion potential] ${ }^{1} \mathrm{M}_{I K}$, need be constant, but $\mathrm{M}_{I K}$, remains continuous 35 across all interfaces that have reached equilibrium [1]".

Because the system under study is non-conservative, we use the entropy-production formulation stated in the form of the Clausius-Duhem [2] inequality. In particular we will consider natural isothermal processes, according to the definition proposed by Planck [3] and adopted by several authors, including Guggenheim [4].

40 For such cases the Clausius-Duhem inequality reduces to the Clausius-Planck inequality 2 .

The dissipation-rate density is the difference between the external power expenditure and the rate of change of free energy of the system per unit volume. It includes contributions from the transport of chemical species and that of charges.

45 It may incorporate mechanical dissipation typical of viscous and plastic materials, however, the treatment of such constitutive behaviors is not included in the finite formulation below.

The thermodynamic framework is constructed along the lines of theories for-

${ }^{1}$ In this paper, we use $\varpi_{i}$ as notation instead of the Larché-Cahn notation $M_{i K}$. The second dependent species $K$ is treated explicitly. 
mulated by Larché and Cahn [1], Truesdell [5], and Ortiz [6]. It also borrows from more recent application to the analysis of electrochemical systems, in particular Li-ion batteries, proposed by Bower 7] 8, Anand 9, Suo [10, Wu 11. As observed by Larché and Cahn 1, in many cases the mutual interaction between stress and diffusion cannot be ignored, therefore it will solve the equations of mechanical equilibrium and diffusion simultaneously. Furthermore, our 55 model extends previous work by accounting for electrostatic forces and multiple charged mobile-species. This includes the internal electric field induced by the transport and distribution of ions and vacancies in order for the system to maintain local electroneutrality. The nonlinear theory of electro-elasticity cast into the Coleman-Noll formalism was originally proposed by Toupin [12, fol${ }_{60}$ lowed by several other contributions, including papers by Ogden and coauthors (see[13 for a literature review). We demonstrate the electro-chemo-mechanical applications of our model by presenting simulations of ambipolar diffusion and kinetic demixing (as defined in 14)-both being relevant phenomena in SOFCs. Kinetic demixing (i.e., the production of non-homogeneous compositions and defect populations due to mobility differences between species) can lead to interfacial segregation. For example, some chemistries and extreme operating conditions will be shown to produce demixing at the surfaces of SOFCs' cathodes. Under operation, SOFC electrodes experience electrical biases that can redistribute their charged defects (e.g. oxygen and cation vacancies [15]). While

70 the oxygen vacancy rapidly moves in response to this bias, cation defects, due in large part to their much lower mobility in these systems, migrate very slowly to establish a new equilibrium. Over time, a cation rich surface layer is often observed to develop in many of the advanced cathodes, blocking the critical oxygen reduction reaction and thus reducing SOFC power [16, 17, 18, 19].

75 With changes in the oxidation state of the electrode (i.e., vacancy content), the relative amount of cation segregation has been shown to be modified [15. In extreme conditions, this may even result in decomposition of the electrode material [20. In another recent example, the oxidation state of the electrode has been controlled to intentionally segregate catalytically active cations from the 80 bulk oxide to the surface, with corresponding improvements in SOFC power [21. Elastic and electrostatic interactions of dopants with the surrounding lattice have been described as the driving forces for segregation on perovskite compounds of the type $\mathrm{Ln}_{1-x} \mathrm{D}_{x} \mathrm{MnO}_{3}$ (host cation $\mathrm{Ln}=\mathrm{La}$, Sm, dopant $\mathrm{D}=$ $\mathrm{Sr}, \mathrm{Ca}, \mathrm{Ba})$ [15] which are among the most commonly used SOFC cathode85 materials. Elastic energy differences are created by differences in the atomic volume of dopants compared to the host cations (La, Sm). Gradients in elastic energy density can affect the stability of cathode surfaces.

Atomistic studies also provide insight for some experimentally observed demixing phenomena [15, 22. Kinetic demixing has also been investigated at the con90 tinuum level for oxide solid solutions in which oxygen ions can be regarded as an immobile species 23, 24. However, these continuum approaches do not account for stress effects on diffusion and segregation of species. Stress driven kinetic demixing and decomposition has been treated by Dimos and co-authors 25]. However, a combined electro-chemo-mechanics is necessary to model complex 
dynamics of these multifunctional materials under a variety of external conditions and microstructures. The rigorous continuum-model proposed herein is amenable for numerical treatment and for the investigation of a wide range of technologically relevant systems in a rigorous quantitative manner. Central to the formulation is the derivation of a diffusion potential for each independent 100 species-the gradients of which drive mass transport. Below, we simplify the formulation by assuming that the diffusion potential can be written as a sum of the chemical, electrostatic, and stress potentials and adopt a mixed finite-element method [26].

Three example applications have been selected for demonstration of the formu-

The range of applications that can be approached goes well beyond the one proposed here and can be further extended in a straightforward manner. The development of this model has been motivated by the need for a rigorous and robust tool to solve a variety of problems in the field of energy storage systems.

This article is organized as follows: Sec. 2 introduces the general theory and its finite element formulation; 3 presents the constitutive laws for the transport of ionic species in an elastic material; Sec. 4 illustrates applications to the solution of ambipolar and kinetic demixing problems and to a case of mechanically

\footnotetext{
${ }^{2}$ Larché and Cahn's work mostly focused on the effect of self-stress, i.e., stress induced by non-homogeneous concentration. In 27. Larché and Cahn took into account problems, which require "the simultaneous solution of the equations of elasticity and those of chemical equilibrium". However, they were able to decouple the chemo-mechanical problem under the assumption of constant diffusion potential. The use of numerical methods permits solutions to a fully coupled electrochemical-mechanical problem with external applied loads.
} 
detailed in the Supplementary contents . A glossary of symbols can be found in Appendix A.

\section{Thermodynamic framework}

We will develop a system of differential equations by considering the variations undergone by a continuous body, having reference configuration $\mathcal{B} \subset \mathbb{R}^{3}$. The motion of the body is described by a time-dependent deformation mapping $\chi: \mathcal{B} \times[a, b] \rightarrow \mathbb{R}^{3}$, where $[a, b]$ is the time interval elapsed during the motion. The variations will include changes in the deformation, concentration of the various chemical species, and the charge of the chemical species. We call this an "electro-chemo-mechanical" variation.

Let $c_{i}$ denote the total number of moles of the chemical species $i$ per unit reference volume $V_{0}$. Changes in $c_{i}$ in a part $\mathcal{P}$ are brought about by the diffusion of the species across its boundary $\partial \mathcal{P}$. The diffusion is characterized by a flux $\mathbf{J}_{i}(X, t)$, i.e. the number of moles of the species entering $\mathcal{P}$ across $\partial \mathcal{P}$, 145 measured per unit area per unit time. ${ }^{3}$

To simplify the form of the equations that follow, all system variables will be measured with respect to an initial reference state with volume $V_{0}$ and not in the electro-chemo-mechanical varied state (the Eulerian state). The results are independent of this choice of frame.

${ }_{150}$ Let $\mathcal{P}$ be an arbitrary part of the reference body $\mathcal{B}$ with $\mathbf{N}$ the outward unit normal on the boundary $\partial \mathcal{P}$ of $\mathcal{P}$. In the finite element formulation that follows, the part $\mathcal{P}$ will correspond to the finite element. Let $c_{i}$ denote the total number of moles of the chemical species $i$ per unit reference volume $V_{0}$. Changes in $c_{i}$ in a part $\mathcal{P}$ are brought about by diffusive processes. Each diffusive process 155 is characterized by the integral of fluxes $\mathbf{J}_{i}(X, t) \cdot \mathbf{N}$, (i.e. $\mathbf{J}_{i}(X, t) \cdot \mathbf{N}$ is the number of moles of each species $i$ entering $\mathcal{P}$ normal to $\partial \mathcal{P}$, measured per unit area per unit time.)

We shall write the local form of the mass balance for the species $i t^{4}$

$$
\dot{c}_{i}=-\operatorname{Div} \mathbf{J}_{i}
$$

\footnotetext{
${ }^{3}$ Notation: $\nabla$ and Div denote the gradient and divergence with respect to the material point in the reference configuration; a superposed dot denotes the material time-derivative. Vectors, second and fourth order tensors are marked with bold and blackboard bold symbols, to distinguish them from scalar values. Throughout, we assume $\mathbf{F}^{e-1}=\left(\mathbf{F}^{e}\right)^{-1}$ and $\mathbf{F}^{e-T}=$ $\left(\left(\mathbf{F}^{e}\right)^{-1}\right)^{T}$

${ }^{4}$ The rate of change of species $i$ in $\mathcal{P}$ is given by

$$
\frac{d}{d t} \int_{\mathcal{P}} c_{i} d V=-\int_{\partial \mathcal{P}} \mathbf{J}_{i} \cdot \mathbf{N} d A
$$

Bringing the time derivative inside the integral and applying the divergence theorem,

$$
\int_{\mathcal{P}}\left(\dot{c}_{i}+\operatorname{Div} \mathbf{J}_{i}\right) d V=0
$$

which leads to the local form of the mass balance for the species $i$.
} 
and analogously the balance of charge

$$
\dot{q}_{i}=-\operatorname{Div} \mathbf{J}_{q_{i}}
$$

where $q_{i}$ the charge of species $i$ per unit volume and $\mathbf{J}_{q_{i}}$ the flux of charges 160 across $\partial \mathcal{P}$ per unit area per unit time.

We will also assume that the body is in local equilibrium so that any gradients of intensive variables are negligible within $\mathcal{P}$.

$$
\begin{aligned}
\frac{d}{d t} \int_{\mathcal{P}} U d V & =-\int_{\partial \mathcal{P}} \mathbf{J}_{Q} \cdot \mathbf{N} d A+\int_{\mathcal{P}} Q d V+\int_{\mathcal{P}} \mathbf{B} \cdot \dot{\chi} d V+\int_{\partial \mathcal{P}} \mathbf{P N} \cdot \dot{\chi} d A+ \\
& -\sum_{i} \int_{\partial \mathcal{P}} \varpi_{i} \mathbf{J}_{i} \cdot \mathbf{N} d A-\sum_{i} \int_{\partial \mathcal{P}} \phi \mathbf{J}_{q_{i}} \cdot \mathbf{N} d A
\end{aligned}
$$

where $U$ is the internal energy per unit of $\mathcal{P}$ 's undeformed volume; $Q$ is the distributed heat source per unit of volume; $\mathbf{J}_{Q}$ is the outward heat flux; $\mathbf{B}$ is the body force per unit volume; ${ }^{5} \mathbf{P}$ is the first Piola-Kirchhoff stress tensor ${ }^{6} \varpi_{i}$ is the diffusion potential of the $i^{t h}$ chemical species; and $\phi$ is the electropotential. The last two terms in Eq. (3) are the chemical and the electrostatic work performed on $\mathcal{P}$. The fourth term is the mechanical work carried out by the traction $\mathbf{T}=\mathbf{P N}$ on the surface $\partial \mathcal{P}$, and the third term is the work performed by any external field via body forces which are conjugate to the deformation 7 . All of the above are the rate of total work transferred to $\mathcal{P}$. The total heat is composed of the first two terms in Eq. (3).

\footnotetext{
${ }^{5}$ In the proposed application $\mathbf{B}$ will be taken as zero. No gravitational effects are considered, and electrostatic forces are not treated as body forces.

A different approach to electromechanical coupling takes into account electric forces into the linear momentum balance 13. Those forces are included in the form of body forces or by defining a total stress, as the sum of the classical mechanical stress and the Maxwell stress. Several contributions using this approach can be found in recent literature in applications to elastomeric and polymeric materials wherein electric field produce very large deformations. The key point is that the mechanical properties of these materials can be changed rapidly and reversibly by externally applied electric fields.

Our treatment of electrostatic forces is limited to their impact on the transport of ionic species. We neglect their effect on the mechanical properties of the material and, therefore, assume negligible the contribution of the Maxwell stress in the equilibrium equation.

${ }^{6}$ The Piola-Kirchhoff stress tensor $\mathbf{P}$ is related to the Cauchy stress tensor $\boldsymbol{\sigma}$ via the Piola transformation

$$
\mathbf{P}=J \boldsymbol{\sigma} \mathbf{F}^{-T}
$$

with $J=\operatorname{det}(\mathbf{F})$ being the determinant of the deformation gradient. The Cauchy stress is defined in the current (or deformed) configuration, while the Piola-Kirchhoff stress is defined in the reference one. We make use of the Piola-Kirchhoff stress in compatibility with the other quantities expressed in the reference configuration. In classical linear mechanics the distinction between reference and Eulerian configuration is not taken into account, and the Cauchy stress is the most commonly used stress quantity.

7 If there were fields that could perform work through variations in any other quantity (such as dipoles, magnetic moments, total charge) within $\mathcal{P}$, then these should be included in Eq. (3). We ignore such fields in this formulation. If the fields were included, they would appear in the coupled differential equations that are derived below in a manner analogous to B.
} 
The second law of thermodynamics for irreversible systems is stated in the form known as Clausius-Duhem inequality

$$
\frac{d}{d t} \int_{\mathcal{P}} s d V-\int_{\mathcal{P}} \frac{\dot{Q}}{T} d V+\int_{\partial \mathcal{P}} \frac{\mathbf{J}_{Q} \cdot \mathbf{N}}{T} d A \geq 0
$$

with $s$ being the entropy per unit of undeformed volume and $T$ the absolute temperature.

In general, the appropriate free energy density for $\mathcal{P}$ depends on which of each conjugate state variable pair are allowed to vary in the electro-chemo-mechanical variation. The free energy density is constructed by performing Legendre transform of the internal energy density $U$ with those conjugate pairs for which the fixed parameter is the intensive state variable. Cahn and Larché used the fixed stress generalization of the Gibbs free energy [28, 1], which, in our notation reads

$$
\Gamma\left(\mathbf{P}, c_{i}, T\right)=U\left(\mathbf{F}, c_{i}, T\right)-s T-\mathbf{P}: \mathbf{F}
$$

with the deformation gradient $\mathbf{F}$ being defined as $\mathbf{F} \equiv \nabla \boldsymbol{\chi}$. Other appropriate work conjugate variables can be used in place of stress $\mathbf{P}$ and the deformation 175 F.

For a displacement-based finite element model of isothermal systems, the free energy density must allow adjacent elements to exchange mechanical work at a constant temperature. Furthermore, to study the transport of ionic species, we construct a free energy density which takes the electropotential as independent variable. It is derived by a Legendre transform of the internal energy as follows

$$
\Upsilon\left(\chi, c_{i}, \phi, T\right)=\inf _{q_{i}}\left[U\left(\chi, c_{i}, q_{i}, s\right)-s T-\sum_{i} \phi q_{i}\right]
$$

The free energy density $\Upsilon$ can be regarded as analogous to the 'grand potential density' (Eq.41 [29]), in the way Leo and Sekerka treat the chemical potential, rather than concentration, as independent variable. From the charge balance (Eq. 22) and applying the divergence theorem we can write

$$
\frac{\partial}{\partial t} \int_{\mathcal{P}} \sum_{i} \phi q_{i} d V=\sum_{i} \int_{\mathcal{P}} \dot{\phi} q_{i} d V-\sum_{i} \int_{\partial \mathcal{P}} \phi \mathbf{J}_{q_{i}} \cdot \mathbf{N} d A
$$

Multiplying the entropy imbalance Eq. (5) by $T$ and subtracting the result from the energy balance Eq. (3), and taking into account Eq. (8) yields the free energy imbalance of $\Upsilon$

$$
\begin{aligned}
& \frac{d}{d t} \int_{\mathcal{P}} \Upsilon d V \leq \\
& \int_{\mathcal{P}} \mathbf{B} \cdot \dot{\chi} d V+\int_{\partial \mathcal{P}} \mathbf{P N} \cdot \dot{\chi} d A-\sum_{i} \int_{\partial \mathcal{P}} \varpi_{i} \mathbf{J}_{i} \cdot \mathbf{N} d A-\sum_{i} \int_{\mathcal{P}} \dot{\phi} q_{i} d V
\end{aligned}
$$

The right hand side of Eq. (9) represents the power expended on $\mathcal{P}$ by the material surrounding $\mathcal{P}$. 
Bringing the time derivative inside the integral and applying the divergence theorem for $\mathcal{P}$

$$
\begin{array}{r}
\int_{\mathcal{P}}\left(\dot{\Upsilon}-\mathbf{B} \cdot \dot{\chi}-\operatorname{Div} \mathbf{P} \cdot \dot{\chi}-\mathbf{P}: \nabla \dot{\chi}+\sum_{i} \varpi_{i} \operatorname{Div} \mathbf{J}_{i}+\sum_{i} \mathbf{J}_{i} \cdot \nabla \varpi_{i}+\right. \\
\left.+\sum_{i} \int_{\mathcal{P}} \dot{\phi} q_{i}\right) d V \leq 0
\end{array}
$$

Assuming force balance

$$
\operatorname{Div} \mathbf{P}+\mathbf{B}=\mathbf{0}
$$

and inserting mass balance (Eq. (1)) into Eq. 10p

$$
\dot{\Upsilon}-\mathbf{P}: \dot{\mathbf{F}}-\sum_{i} \varpi_{i} \dot{c}_{i}+\sum_{i} \mathbf{J}_{i} \cdot \nabla \varpi_{i}-\sum_{i} \dot{\phi} q_{i} \leq 0
$$

The rate of dissipation density is the difference between the external power expenditure and the rate of change of free energy density of the element $\mathcal{P}$. From Eq. (12):

$$
\mathcal{D}=\mathbf{P}: \dot{\mathbf{F}}+\sum_{i} \varpi_{i} \dot{c}_{i}-\sum_{i} \mathbf{J}_{i} \cdot \nabla \varpi_{i}+\sum_{i} \dot{\phi} q_{i}-\dot{\Upsilon} \geq 0
$$

\section{Constitutive equations}

\subsection{Elastic lattice material}

We consider a multiplicative decomposition of the deformation gradient.

An intercalating species will be treated as a substitutional atom according to

185 Larché and Cahn model [30, and it will be be assumed that the intercalating species has a fixed valence. Treating electrochemical intercalation in this way, the fraction of intercalation sites that are occupied by vacancies will vary from 0 to $c_{\max }$. The deformation that arises from Vegard's law (i.e., the strain associated with the concentration of intercalating atoms) is the anelastic deformations $F^{a}$, while the recoverable lattice distortion is the elastic deformation $F^{e}$.

We assume a multiplicative decomposition of the total deformation gradient

$$
\mathbf{F}=\mathbf{F}^{e} \mathbf{F}^{a}
$$

according to elastic-anelastic kinematics. The anelastic deformation gradient

$$
\mathbf{F}^{a}=\mathbf{F}^{a}\left(c_{i}\right)
$$

is defined as function of the local concentration of the intercalating species 8 . In the case of isotropic Vegard's law volumetric expansion (similarly with isotropic

\footnotetext{
8 It follows that the elastic deformation is also a function of the species concentration $\mathbf{F}^{e}\left(\chi, c_{i}\right)=\mathbf{F}(\chi) \mathbf{F}^{a-1}\left(c_{i}\right)$
} 
thermal expansion) Eq. (15) is an extension of Eq. (4.1) in [1] to nonlinear kinematics. It is also related to the partial molar volume of the intercalating species with respect to the host lattice. We restrict the examples herein to the case of an isotropic volumetric expansion of the form 9

$$
\mathbf{F}^{a}\left(c_{i}\right)=\left(1+\frac{1}{\rho_{h}} \sum_{i} \beta_{i} c_{i}\right) \mathbf{I}
$$

where the molar volume of the hosting compound is $\rho_{h}$ (in number of moles per unit of undeformed volume).

Let us consider the following generic forms for the free energy density, the first Piola-Kirchhoff stress tensor, the diffusion potential and the electropotential

$$
\begin{aligned}
& \Upsilon=\Upsilon\left(\mathbf{F}^{e}, c_{i}, \phi\right) \\
& \mathbf{P}=\mathbf{P}\left(\mathbf{F}^{e}, c_{i}, \phi\right) \\
& \varpi_{i}=\varpi_{i}\left(\mathbf{F}^{e}, c_{i}, \phi\right) \\
& \phi=\phi\left(\mathbf{F}^{e}, c_{i}, \phi\right)
\end{aligned}
$$

Substituting the constitutive equations above into the free-energy imbalance Eq. (12) leads to the following

$$
\begin{aligned}
& 0 \geq \\
& \frac{\partial \Upsilon\left(\mathbf{F}^{e}, c_{i}, \phi\right)}{\partial \mathbf{F}^{e}}: \dot{\mathbf{F}}^{e}+\sum_{i}\left(\frac{\partial \Upsilon\left(\mathbf{F}^{e}, c_{i}, \phi\right)}{\partial c_{i}} \dot{c}_{i}\right)+\frac{\partial \Upsilon\left(\mathbf{F}, c_{i}, \phi\right)}{\partial \phi} \dot{\phi}+ \\
& -\mathbf{P}:\left(\dot{F}^{e} \mathbf{F}^{a}+\mathbf{F}^{e} \dot{\mathbf{F}}^{a}\right)-\sum_{i} \varpi_{i} \dot{c}_{i}+\sum_{i} \mathbf{J}_{i} \cdot \nabla \varpi_{i}-\sum_{i} \dot{\phi} q_{i}
\end{aligned}
$$

In order for inequality Eq. (22) to hold for arbitrary combinations of the independent variables $\left(\mathbf{F}^{e}, c_{i}, \phi\right)$, the coefficients of Eq. (22) must vanish. We are therefore led to the thermodynamic restriction that relates the free energy

9 The coefficients $\beta_{i}$ correspond to the ratio between the lattice parameter of the intercalating species $i$ and the one of the hosting compound. The relative volume change upon intercalation can be computed as the determinant of the anelastic deformation tensor. In linear kinematics it can be approximated as follows

$$
\frac{V+d V}{V}=\operatorname{det}\left(\mathbf{F}^{a}\right)=\left(1+\frac{1}{\rho_{h}} \sum_{i} \beta_{i} c_{i}\right)^{3} \rightarrow 1+3 *\left(\frac{1}{\rho_{h}} \sum_{i} \beta_{i} c_{i}\right)
$$

such approximation is valid in the limit $\beta_{i} \rightarrow 0$. The factor $3 \beta_{i} / \rho_{h}$ corresponds to the partial molar volume of the intercalating species with respect to the hosting compound. 
density $\Upsilon$ to the stress $\mathbf{P}$ the diffusion potential $\varpi_{i}{ }^{10}$ and the electropotential $\phi$

$$
\begin{aligned}
\mathbf{P} & =\frac{\partial \Upsilon\left(\mathbf{F}^{e}, c_{i}, \phi\right)}{\partial \mathbf{F}^{e}} \mathbf{F}^{a-T} \\
\varpi_{i} & =\frac{\partial \Upsilon\left(\mathbf{F}^{e}, c_{i}, \phi\right)}{\partial c_{i}}-\frac{\beta_{i}}{\rho_{h}} \operatorname{Tr}\left(\mathbf{F}^{e T} \mathbf{P}\right) \\
q_{i} & =\frac{\partial \Upsilon\left(\mathbf{F}^{e}, c_{i}, \phi\right)}{\partial \phi}
\end{aligned}
$$

In the literature this procedure is sometimes referred to as the Coleman-Noll procedure [31].

For the derivatives with respect to $c_{i}$, only those $c_{i}$ which are independently variable are included. For example, a network constraint (conservation of a particular lattice position) produces an additional equation of the form:

$$
c_{i}+c_{j}=\text { constant }_{i j}
$$

In this work, the constraints will be inserted into $\Upsilon$ explicitly; for example: $\Upsilon\left(\mathbf{P}, c_{1}, c_{2}, \ldots, c_{j-1}\right.$, constant $\left._{i j}-c_{i}, c_{j+1}, \ldots, q_{i}\right)$.

The dissipation in Eq. (13) reduces to

$$
\mathcal{D}=-\sum_{i} \mathbf{J}_{i} \cdot \nabla \varpi_{i} \geq 0
$$

Eq. (28) consists of the energy density dissipated in transport of chemical species. In Eq. 28) extra terms will arise in presence of mechanical dissipation, for example in plastic or viscous materials.

The inequality in Eq. 287 demands that the projection of the fluxes onto the gradients of their conjugate fields is negative.

${ }_{205}$ We now seek to find a description of the rate problem, i.e., finding the rate of electro-chemo-mechanical variation of $\mathcal{P}$ given its boundary conditions.

To develop the finite element formulation for an electro-chemo-mechanical system, we will choose a less general system, with a free energy density that can be decomposed as follows:

$$
\Upsilon\left(\mathbf{F}^{e}, c_{i}, \phi\right)=\Upsilon^{M}\left(\mathbf{F}^{e}, c_{i}\right)+\Upsilon^{E C}\left(c_{i}, \phi\right)
$$

\footnotetext{
${ }^{10}$ Given the following identities

$$
\frac{\partial}{\partial n_{i}}=\frac{\partial}{\partial c_{i}} \frac{\partial c_{i}}{\partial n_{i}}=\frac{\partial}{\partial c_{i}} \frac{1}{V_{0}}
$$

the first term in the state equation Eq. 25 of the diffusion potential $i$ (in the case of transport of species uncoupled from mechanical and electrostatic effects) is compatible with the classical definition of the chemical potential as derivative of the internal energy (integrated over the reference volume) with respect to the number of moles $n_{i}$ of species $i$; where entropy, volume and number of moles of species $k \neq i$ are held constant. The second term in Eq. 25 corresponds to the stress dependent component of the diffusion potential introduced by Larché and Cahn in [30. If we used the partial molar volume, instead of the relative lattice parameter $\beta_{i}$, in the definition of the anelastic deformation a $\frac{1}{3}$ factor would appear in the expression.
} 
The function $\Upsilon^{M}$ determines the elastic response of the material, $\Upsilon^{E C}$ represents the electrochemical free energy density.

In the presence of only anelastic deformation $\left(\mathbf{F}^{e}=\mathbf{I} ; \quad \mathbf{F}=\mathbf{F}^{a}\right)$, we assume that the mechanical free energy vanishes

$$
\Upsilon^{M}\left(\mathbf{F}^{e}=\mathbf{I}, c_{i}\right)=0
$$

Assuming that there is no residual stress, any choice of mechanical free energy density requires that a global minimum appears when there is no elastic deformation $\left(\mathbf{F}^{e}=\mathbf{I}\right.$ and $\left.\mathbf{F}=\mathbf{F}^{a}\right)$, in other words, the mechanical energy minimizing state is characterized by stress-free kinematics.

Any concentration gradient that develops will produce incompatible inelastic deformations which generate stress.

Objectivity (frame indifference) of $\mathcal{P}$ 's energy imposes a restriction on the dependence of the elastic energy density on the elastic strain tensor

$$
\Upsilon^{M}\left(\mathbf{F}^{e}, c_{i}\right) \equiv \Upsilon^{M}\left(\mathbf{C}^{e}, c_{i}\right)
$$

with $\mathbf{C}^{e}=\mathbf{F}^{e T} \mathbf{F}^{e}$ being the elastic right Cauchy-Green strain tensor 32. In other words, rigid body motion does not change the energy density of the element $\mathcal{P}$.

Given the form of the free energy density Eq. (29), the $i^{\text {th }}$ diffusion potential of Eq. 25) is decomposed into the sum of mechanical, and electrochemical terms

$$
\begin{aligned}
\varpi_{i}\left(\mathbf{C}^{e}, c_{i}\right) & =\frac{\partial \Upsilon^{M}\left(\mathbf{C}^{e}, c_{i}\right)}{\partial c_{i}}+\frac{\partial \Upsilon^{E C}\left(c_{i}, \phi\right)}{\partial c_{i}}-\frac{\beta_{i}}{\rho_{h}} \operatorname{Tr}\left(\mathbf{F}^{e T} \mathbf{P}\right) \\
& =\mu_{i}^{M}\left(\mathbf{C}^{e}, c_{i}\right)+\mu_{i}^{E C}\left(c_{i}, \phi\right)
\end{aligned}
$$

with the following definitions of mechanical and electrochemical components of the diffusion potential

$$
\begin{aligned}
& \mu_{i}^{M}\left(\mathbf{C}^{e}, c_{i}\right)=\frac{\partial \Upsilon^{M}\left(\mathbf{C}^{e}, c_{i}\right)}{\partial c_{i}}-\frac{\beta_{i}}{\rho_{h}} \operatorname{Tr}\left(\mathbf{F}^{e T} \mathbf{P}\right) \\
& \mu_{i}^{E C}\left(c_{i}, \phi\right)=\frac{\partial \Upsilon^{E C}\left(c_{i}, \phi\right)}{\partial c_{i}}
\end{aligned}
$$

3.2. State functions for transport of neutral species within an elastic material having a network constraint

Let us consider first the treatment of transport of an uncharged chemical species within an imposed network constraint; this is, for instance, the case of the diffusion by vacancy exchange mechanism.

In the case of non-ideal solution conditions and constant temperature, the electrochemical free energy per unit of reference volume for the system under study takes the form 33

$$
\Upsilon^{E C}\left(c_{i}, c_{V_{i}}\right)=c_{i} R T \ln c_{i}+c_{V_{i}} R T \ln c_{V_{i}}+\Upsilon_{I I}^{E C}\left(c_{i}, c_{V_{i}}\right)
$$


where $c_{i}$ represents the number of moles per unit of reference volume of the diffusing species and $c_{V_{i}}$ is the molar density of the corresponding vacancies; ${ }_{225} \Upsilon_{I I}^{E C}$ is the excess of free energy caused by the interactions among intercalated species.

The network constraint results in the following condition

$$
\dot{c}_{i}+\dot{c}_{V_{i}}=0
$$

It follows that

$$
c_{i}+c_{V_{i}}=c_{i_{\max }}=\text { constant }
$$

i.e. the total number of sites $c_{i_{\max }}$ is conserved. Furthermore we can express the molar density of vacancies as $c_{V_{i}} \equiv c_{V_{i}}\left(c_{i}\right)$.

The electrochemical potential $\mu^{E C}$, derived from Eq. 35 according to Eq. 25. takes the form (as in Eq. 5.16 in [1])

$$
\begin{aligned}
\mu_{i}^{E C}\left(c_{i}\right) & =\frac{\partial \Upsilon^{E C}}{\partial c_{i}}+\frac{\partial \Upsilon^{E C}}{\partial c_{V_{i}}} \frac{\partial c_{V_{i}}}{\partial c_{i}} \\
& =R T \ln c_{i}-R T \ln c_{V_{i}}+\frac{\Upsilon_{I I}^{E C}}{\partial c_{i}}-\frac{\Upsilon_{I I}^{E C}}{\partial c_{V_{i}}} \\
& =R T \ln \frac{c_{i}}{c_{i_{\text {max }}}-c_{i}}+R T \ln \frac{\gamma_{i}}{\gamma_{V_{i}}}
\end{aligned}
$$

In Eq. 38 we assume a definition of the excess of free energy such that

$$
\begin{aligned}
& \frac{\Upsilon_{I I}^{E C}}{\partial c_{i}}=R T \ln \gamma_{i} \\
& \frac{\Upsilon_{I I}^{E C}}{\partial c_{V_{i}}}=R T \ln \gamma_{V_{i}}
\end{aligned}
$$

The activity coefficient $\bar{\gamma}_{i}\left(c_{i}\right)=\frac{\gamma_{i}}{\gamma_{V_{i}}}$ represents the deviation from the infinite dilute solution condition. In non-isothermal systems $\bar{\gamma}_{i}$ will also be dependent on the temperature.

We shall consider a Neo-Hookian energy functional as a suitable form for the elastic free energy density 32

$$
\Upsilon^{M}\left(I_{1}\left(\mathbf{C}^{e}\right), J\left(\mathbf{C}^{e}\right), c_{i}\right)=\frac{1}{2} \lambda(\log J)^{2}-G \log J+\frac{1}{2} G\left(I_{1}-3\right)
$$

In Eq. 41) we make use of the definitions of the Jacobian ${ }^{11} J=\sqrt{\operatorname{det}\left(\mathbf{C}^{e}\right)}$, and of the first invariant of the elastic right Cauchy-Green strain tensor $I_{1}=\operatorname{Tr}\left(\mathbf{C}^{\mathbf{e}}\right)$. The first Lamé constant $\lambda$ and the shear modulus $G$ in Eq. (41) can be defined

\footnotetext{
${ }^{11}$ Not to be confused with $\mathbf{J}$, a vector indicating the flux. A prescribed flux on the boundary will be represented with the scalar valued quantity $\bar{J}$.
} 
as concentration dependent elastic properties of the material according to the following constitutive law

$$
\begin{array}{r}
\lambda\left(c_{i}\right)=\lambda_{0}+\lambda_{1}\left(c_{i}-c_{i_{0}}\right) \\
G\left(c_{i}\right)=G_{0}+G_{1}\left(c_{i}-c_{i_{0}}\right)
\end{array}
$$

where $c_{i_{0}}$ is the reference concentration at which $\lambda_{0}$ and $G_{0}$ are defined.

The first Piola-Kirchhoff stress can be derived from Eq. (41) according to Eq. (24)

$$
\mathbf{P}=\frac{\partial \Upsilon^{M}}{\partial \mathbf{F}^{e}} \mathbf{F}^{a-T}=\left[(\lambda \log J-G) \mathbf{F}^{e-T}+G \mathbf{F}^{e}\right] \mathbf{F}^{a-T}
$$

Analogously the elasticity tensor is computed as follows

$$
\mathbb{C}=\frac{\partial^{2} \Upsilon^{M}}{\partial \mathbf{F} \partial \mathbf{F}} \mathbf{F}^{a-1} \frac{\partial^{2} \Upsilon^{M}}{\partial \mathbf{F}^{e} \partial \mathbf{F}^{e}} \mathbf{F}^{a-T}=\mathbf{F}^{a-1} \mathbb{C}^{e} \mathbf{F}^{a-T}
$$

12

$$
\mathbb{C}^{e}=\lambda \mathbf{F}^{e-T} \otimes \lambda \mathbf{F}^{e-T}-(\lambda \log J-G) \mathbf{F}^{e-1} \odot \lambda \mathbf{F}^{e-1}+G \mathbb{I}
$$

By making use of Eq. (38), in absence of electrostatic effects, the diffusion potential in Eq. 32 takes the form

$$
\begin{aligned}
\varpi\left(\mathbf{C}^{e}, c_{i}\right) & =\frac{\partial \Upsilon^{M}\left(\mathbf{C}^{e}, c_{i}\right)}{\partial c_{i}}+\frac{\partial \Upsilon^{E C}\left(c_{i}\right)}{\partial c_{i}}-\beta_{i} \operatorname{Tr}\left(\mathbf{F}^{e T} \mathbf{P}\right) \\
& =\frac{1}{2} \lambda_{1}(\log J)^{2}-G_{1} \log J+\frac{1}{2} G_{1}\left(I_{1}-3\right)+ \\
& +R T \ln \frac{c_{i}}{c_{i_{\max }}-c_{i}}+R T \ln \bar{\gamma}_{i}-\frac{\beta_{i}}{\rho_{h}} \operatorname{Tr}\left(\mathbf{F}^{e T} \mathbf{P}\right)
\end{aligned}
$$

This is a generalization of E.(5.16) of [1] to nonlinear kinematics.

If the elastic properties vary with composition according to Eq. (42), the elasticity tensor $\mathbb{C}^{e}$ in Eq. 45 will be composition dependent, analogously to the open system compliance defined in Reference [1. Because the elasticity tensor $\mathbb{C}$ in Eq. 44 contains the anelastic deformation $\mathbf{F}^{a}$, it remains function of $c_{i}$, even in the case of constant elastic properties. The derivative of the mechanical free energy density with respect to composition

$$
\frac{\partial \Upsilon^{M}\left(\mathbf{C}^{e}, c_{i}\right)}{\partial c}=\frac{1}{2} \lambda_{1}(\log J)^{2}-G_{1} \log J+\frac{1}{2} G_{1}\left(I_{1}-3\right)
$$

\footnotetext{
${ }^{12}$ Operators used in Eq. 45 have the following definition

$$
\begin{array}{r}
(\mathbf{A} \otimes \mathbf{A})_{i j k l}=\mathbf{A}_{i j} \mathbf{A}_{k l} \\
(\mathbf{A} \odot \mathbf{A})_{i j k l}=\frac{1}{2}\left(\mathbf{A}_{i k} \mathbf{A}_{j l}+\mathbf{A}_{i l} \mathbf{A}_{j k}\right)
\end{array}
$$
}

and the 4th-order identity tensor $\mathbb{I}$ is

$$
(\mathbb{I})_{i j k l}=\delta_{i k} \delta_{j l} \quad \mathbb{I} \mathbf{A}=\mathbf{A}
$$


is null in the case elastic properties independent from concentration, namely if

$\lambda_{1}=G_{1}=0$.

\subsection{State functions for transport of multiple ionic species subject to mass and charge balance}

In ionic systems, it is necessary to account for the effects of gradients in the electrostatic potential on the flux of charged species. Consequently, the total 245 driving force for mass transport includes the electrochemical potential and the stress-dependent contribution associated with changes in local molar volume. The electrochemical free energy density for this case will include an additional term respect to Eq. 35] to account for migration effect

$$
\Upsilon^{E C}\left(c_{i}, c_{V_{i}}\right)=c_{i} R T \ln c_{i}+c_{V_{i}} R T \ln c_{V_{i}}+\Upsilon_{I I}^{E C}\left(c_{i}, c_{V_{i}}\right)+z_{i} F c_{i} \phi
$$

$F=e N_{a}$ is the Faraday constant 13 . The ion valence is $z_{i}$.

The diffusion potential for the charged species $i$, in presence of a network constraint on the number of $i$-sites available, is derived from Eq. 25) and can be expressed as

$$
\begin{aligned}
& \varpi_{i}\left(\mathbf{C}^{e}, c_{i}\right)=\frac{\partial \Upsilon^{M}\left(\mathbf{C}^{e}, c_{i}\right)}{\partial c_{i}}+\frac{\partial \Upsilon^{E C}\left(c_{i}, \phi\right)}{\partial c_{i}}-\beta_{i} \operatorname{Tr}\left(\mathbf{F}^{e T} \mathbf{P}\right)= \\
& =\frac{\partial \Upsilon^{M}\left(\mathbf{C}^{e}, c_{i}\right)}{\partial c_{i}}+R T \ln \left(\frac{c_{i}}{c_{i_{\max }}-c_{i}}\right)+R T \ln \bar{\gamma}_{i}+z_{i} F \phi-\frac{\beta_{i}}{\rho_{h}} \operatorname{Tr}\left(\mathbf{F}^{e T} \mathbf{P}\right)
\end{aligned}
$$

with the dependence of the elastic properties on concentration given by the term

$$
\frac{\partial \Upsilon^{M}\left(\mathbf{C}^{e}, c_{i}\right)}{\partial c_{i}}=\frac{1}{2} \lambda_{1}(\log J)^{2}-G_{1} \log J+\frac{1}{2} G_{1}\left(I_{1}-3\right)
$$

In a multi-species diffusion process, the ionic fluxes are computed in the deformed configuration according to

$$
\mathbf{F} \mathbf{J}_{i}=-\frac{M_{i}}{R T} c_{i} \nabla_{\mathbf{x}}\left(\varpi_{i}\right)
$$

or equivalently in reference configuration ${ }^{14}$

$$
\mathbf{J}_{i}=-\frac{M_{i}}{R T} c_{i} \mathbf{C}^{-1} \nabla\left(\varpi_{i}\right)
$$

\footnotetext{
${ }^{13}$ Not to be confused with $\mathbf{F}$, a tensor values variable, representing the deformation gradient.

${ }^{14}$ Notation: the symbol $\nabla_{\mathbf{x}}$ denotes the gradient with respect to the deformed configuration. The following transformation between reference and spatial configuration (coordinate system marked respectively with $\mathbf{X}$ and $\mathbf{x}$ ) applies

- $\nabla_{\mathbf{x}} f=\mathbf{F}^{-T} \nabla f, f$ being a generic scalar function;

- $\mathbf{u}=\mathbf{F U}, \mathbf{u}$ being a vector defined in the spatial configuration and $\mathbf{U}$ the corresponding vector in the material configuration.
} 
assuming that the fluxes are independent; that is there are no cross-correlation terms in the Onsager coefficients (i.e., the flux on one sub-lattice does not affect fluxes on other sub-lattices).

Substituting Eq. (48) into Eq. (50) we obtain an extended form of the NernstPlanck equation, which includes mechanical effects

$$
\begin{aligned}
\mathbf{J}_{i}= & -M_{i}\left[\left(\frac{c_{i_{\max }}}{c_{i_{\max }}-c_{i}}\right) \mathbf{C}^{-1} \nabla c_{i}+z_{i} c_{i} \frac{F}{R T} \mathbf{C}^{-1} \nabla \phi\right. \\
& \left.-\frac{\beta_{i} c_{i}}{R T} \mathbf{C}^{-1} \nabla\left(\mathbf{F}^{e T} \mathbf{P}: \mathbf{I}\right)\right]
\end{aligned}
$$

Here the treatment of electrostatic forces is limited to their impact on the transport of ionic species. We disregard, for instance, their effect on the mechanical properties of the material.

In the literature [34, 35] the condition ${ }^{15}$

$$
\sum_{i} z_{i} \mathbf{J}_{i}=\mathbf{0}
$$
reestablish electroneutrality. However, local polarization is not treated here and treatment of piezoelectric effects (local charge polarization related to the occurrence of electric dipole moments) and electrical bias is left to future publications.

The internal electric field $\mathbf{E}$ with respect to the reference configuration can be computed by inserting the expression for the ionic fluxes Eq. (50) into the charge balance equation Eq. (52) ${ }^{16}{ }^{17}$

$$
\mathbf{E}(\mathbf{X}, t)=\nabla \phi=-\mathbf{C} \frac{R T}{F} \frac{\sum_{i} z_{i} \frac{M_{i}}{R T} c_{i} \mathbf{C}^{-1} \nabla \mu_{i}^{C M}}{\sum_{i} z_{i}^{2} M_{i} c_{i}}
$$

\footnotetext{
${ }^{15}$ For a discussion on the validity of the electroneutrality condition Eq. 52 we refer the reader to Section 11.8 of 35 .

16 The chemo-mechanical component of the diffusion potential appearing in Eq. 53 corresponds to the following definition

$$
\mu_{i}^{C M}\left(\mathbf{C}^{e}, c_{i}\right)=\frac{\partial \Upsilon^{M}\left(\mathbf{C}^{e}, c_{i}\right)}{\partial c_{i}}+R T \ln \left(\frac{c_{i}}{c_{i_{\max }}-c_{i}}\right)+R T \ln \bar{\gamma}_{i}-\frac{\beta_{i}}{\rho_{h}} \operatorname{Tr}\left(\mathbf{F}^{e T} \mathbf{P}\right)
$$

${ }^{17}$ In 53 we made use of the relation $\mathbf{E}=\mathbf{F}^{T} \mathbf{e}$ between the electrostatic field $\mathbf{e}(\mathbf{x}, t)=\nabla_{\mathbf{x}} \phi$ defined in the deformed configuration, the corresponding field $\mathbf{E}(\mathbf{X}, t)=\nabla \phi$ in the Lagrangian configuration (see 13 for the complete derivation).
} 
265 As part of the numerical procedure, the local electric field is computed according to Eq. (53). The gradient of the electrostatic potential is then inserted into the expression of the flux Eq. (51) for each of the charged species. This numerical procedure is repeated at each assembly of the stiffness matrix $\mathcal{K}^{c c}$ (see Eq. (B.15) ) at each iteration in the solution of the nonlinear coupled problem.

270 At convergence, because local charge balance is reached, the local electric field converges to zero.

\section{Example applications}

\subsection{Ambipolar diffusion}

Ambipolar diffusion is an example of coupled transport of charged species. The 275 rate of transport of both species is characterized by an apparent diffusion coefficient (i.e., the ambipolar diffusion coefficient), whose value is bounded by the diffusivities of the individual species involved. Charge neutrality is maintained through an internal electric field which fixes the separation of cations an anions even if their mobilities and driving forces differ. This phenomenon is observed in the case of oxidation or reduction of an oxide or a metal, formation of an oxide film in metal corrosion, mass transport under stress gradients during sintering, diffusional creep, etc. The simultaneous preservation of electrical neutrality and stoichiometry comes from the coupled fluxes of anions and cations from the same source to the same sink. Anion and cation rich regions may form, instead, in

285 the case of solid demixing as presented in the following section.

When the stress effect can be considered negligible and in the limit of a dilute solid-solution the internal electric field of Eq. (53) (written in the spatial configuration) takes the form

$$
\mathbf{e}=-\frac{R T}{F} \frac{\sum_{i} z_{i} D_{i} \nabla_{\mathbf{x}} c_{i}}{\sum_{i} z_{i}^{2} D_{i} c_{i}}
$$

typically used in literature (see for instance [34). By substituting Eq. (54) into the Nernst-Planck equation Eq. 51), it is possible to compute an ambipolar diffusivity, that characterizes combined anion and cation transport. For instance, in the presence of two diffusing species, a cation $\mathrm{A}$ and an ion $\mathrm{B}$ of valence +1 and -1 respectively, the ambipolar diffusivity is: 34

$$
\tilde{D}=\frac{D_{A^{+}} D_{B^{-}}}{D_{A^{+}}+D_{B^{-}}}
$$

The extension of the result in Eq. (55) to a general case (electro-chemo-mechanical diffusion potential and arbitrary number of species, defects, and lattice constraints) is presented in 4.1.1.

The numerical method permits material parameters to be varied and explore 290 the relative weight of the different components of the diffusion potential. To illustrate the numerical method and simplify the discussion, one-dimensional models are presented for a series of physical cases. Three dimensional models with microstructures will be presented elsewhere. 
Boundary conditions

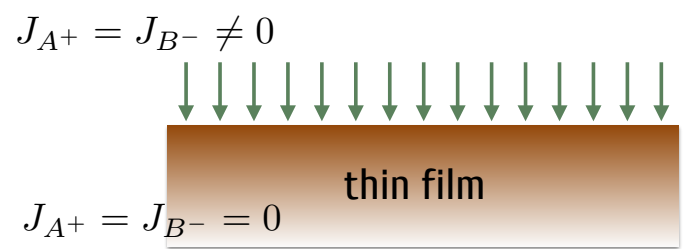

rigid substrate
Initial conditions

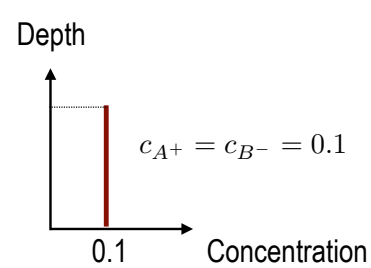

Consider an elastic thin film deposited on a substrate with two species $A$ and $B$ with differing mobility. The film is assumed to be rigidly bounded to the substrate, so that only out-of-plane deformations are allowed, and a plane stress condition is established. Electrostatic effects can be quantified by comparison Suppose the mobility of $B$ is two orders of magnitude smaller than that of $A$. Diffusion is assumed to occur by a vacancy mechanism; therefore there is a network constraint. We will suppose that $A$ and $B$ occupy different lattice positions; so their network constraints are independent. This means that $A$ on the $\alpha$ lattice is always opposite to the flux of $A$. Because $A$ and its vacancy have opposite effective charges, each sub-lattice maintains electroneutrality. Time-independent boundary fluxes $J_{A}$ and $J_{B}$ of equal magnitude are imposed at the interface away from the substrate (top surface in Fig. 11), and $J_{A}=$ 310 $J_{B}=0$ at the substrate interface. Boundary and initial conditions for this set of problems are illustrated in Fig. 1, and a list of input parameters is collected in Tab 1.

All results below are produced by a one-dimensional finite element model. Spatial, temporal, and concentration variables are non-dimensionalized (Time: $\tau \equiv$ $t D_{A} / L^{2}, L$ being the film thickness and Depth: $\xi \equiv X / L$, and Concentration: $\zeta_{i} \equiv$ $c_{i} / c_{i_{\max }}$ where $c_{i_{\max }}$ is the limiting value of the $i^{t h}$ species solubility). Details of the finite element formulation and its numerical solutions are presented in Appendix B and in the online supporting material .

The problem is discretized in space (with 100 finite elements within the film 320 thickness) and in time. To find the solution of the nonlinear coupled problem at each time step, a Newton-Raphson procedure is employed. The electrochemicalmechanical problem to be solved at each iteration of the Newton-Raphson algorithm is given by Eq. B.23) of Appendix B. Constitutive laws for this problem 
can be found in Section 3 and listed again in Section Appendix B

325 The panels in Fig. 2 illustrate the difference between the concentration evolution for charged and neutral species and serve as examples to illustrate the effect of the electrostatic potential in addition to the chemical potentials in coupling the transport of two charged species.

Fig. 2a shows that high-mobility neutral species $A$ has a very small concen330 tration polarization: $\zeta_{A}(\xi, \tau)$ (the normalized concentration field for species $A$ ) is approximately uniform through the film thickness; $\xi$, evolves linearly with time $\tau$ as dictated by the intensity of the external flux. Because the diffusion length is large compared to the film thickness, the steady-state condition appears rapidly. In Fig. 2a (left image), the small non-uniform concentration 335 gradient is not resolved.

Conversely, the contour plot (middle figure in Fig. 2a) for the neutral species $B$ reveals concentration polarization, i.e. the presence of a diffusion front that slowly moves across the sample, as soon as the region above it has reached saturation. The low-mobility species $B$ concentration polarization is illustrated by plotting the concentration evolution at the top an bottom interfaces (right portion of Fig. 2a).

An identical numerical experiment (with two substitutional charged species $A^{+}$ $B^{-}$) is shown in Fig. $2 \mathrm{~b}$.

We will assume that vacancies are created as a Schottky defect; in the Kröger-

345 Vink notation 36] is written as

null $\longrightarrow \mathrm{V}_{\mathrm{A}}^{\prime}+\mathrm{V}_{\mathrm{B}}^{\bullet}$

where $\mathrm{V}_{\mathrm{A}}^{\prime}$ and $\mathrm{V}_{\mathrm{B}}^{\bullet}$ are the respective charged vacancies on the cation $(\mathrm{A})$ and anion (B) sites. The network constraint on the total number of lattice sites available for ions and vacancies is expressed

$$
\begin{aligned}
& c_{\mathrm{A}_{\mathrm{A}}^{\times}}+c_{\mathrm{V}_{\mathrm{A}}^{\prime}}=c_{A_{\max }}=\text { constant } \\
& c_{\mathrm{B}_{\mathrm{B}}^{\times}}+c_{\mathrm{V}_{\mathrm{B}}^{\bullet}}=c_{B_{\max }}=\text { constant }
\end{aligned}
$$

and the corresponding electrochemical potentials take the form

$$
\begin{aligned}
& \mu_{A}^{E C}\left(c_{A}\right)=R T \ln \frac{c_{A}}{c_{A_{\max }}-c_{A}}+R T \ln \bar{\gamma}_{A} \\
& \mu_{B}^{E C}\left(c_{B}\right)=R T \ln \frac{c_{B}}{c_{B_{\max }}-c_{B}}+R T \ln \bar{\gamma}_{B}
\end{aligned}
$$

In this examples we will consider the solution to be ideal, (i.e., $\bar{\gamma}_{A}=\bar{\gamma}_{B}=1$ ). The gradient of the electrostatic potential is computed according to Eq. (53), then inserted into the expression of the flux Eq. (51) for each of the charged species. This procedure is repeated during the assembly of the stiffness matrix $\mathcal{K}^{c c}$ (see Eq. (B.15)) at each iteration in the solution of the nonlinear coupled problem of Eq. (B.23) of Appendix B. The coupling in the transport of the two ionic species is illustrated in the contour plot of Fig. 2b. The effective diffusivity is bounded by $D_{A^{+}}$and $D_{B^{-}}$, but is approximately equal to the lowest diffusivity $D_{B^{-}}$in this case. 

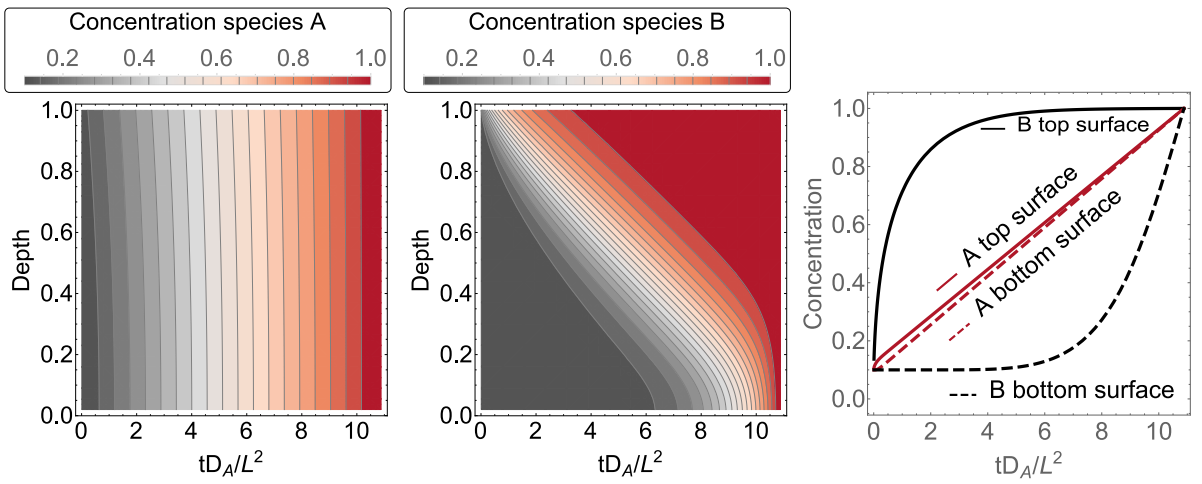

(a) Diffusion of neutral species
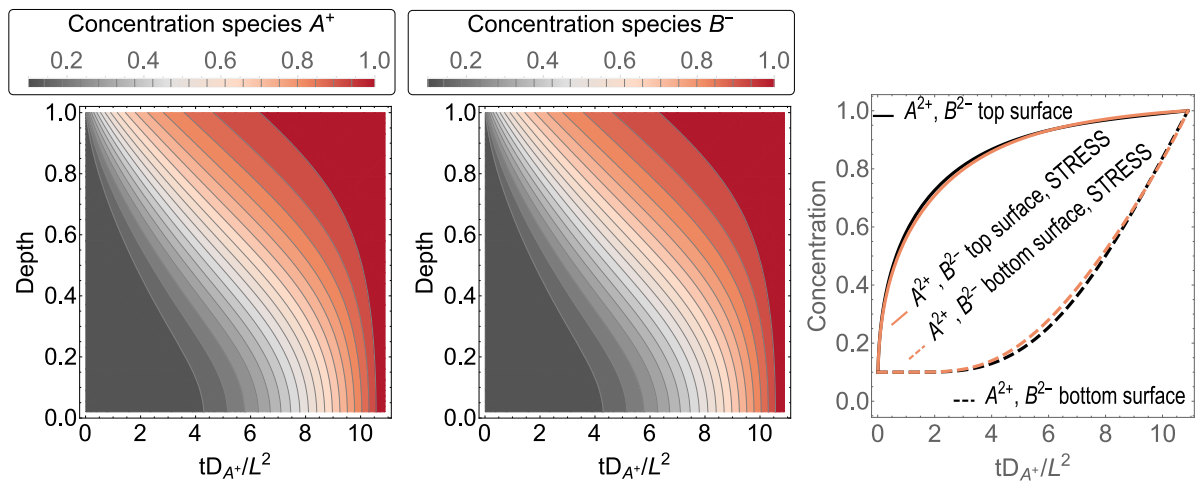

(b) Diffusion of charged species

Figure 2: Comparison of ambipolar diffusion of neutral and charged particles (panels A and B respectively). The mobility of species $A$ is 100 times that of $B$; this emphasizes the effect of the electrostatic coupling. The enforcement of electroneutrality is expressed as a local constraint on the fluxes of cations and ions. The rightmost picture in Fig. 2b (orange line) shows how the presence of stress slightly changes the concentration profile of $A^{+}$and $B^{-}$. However, the electrostatic driving force remains dominant and it couples the transport of the two ions. 
Furthermore, if Vegard strains are introduced along with the charged species, the effect of stress on ambipolar diffusion can be simulated. 
Table 1: Material parameters for problems in Section 4.1.1

\begin{tabular}{ll} 
Input value & Description \\
\hline$F=96485.3365 \mathrm{C} \mathrm{mol}^{-1}$ & Faraday's constant \\
$R=8.314 \mathrm{~J} \mathrm{~K}^{-1} \mathrm{~mol}^{-1}$ & gas constant \\
$T=298 \mathrm{~K}$ & temperature \\
$M_{A}=10^{-15} \mathrm{~m}^{2} \mathrm{~s}^{-1}$ & mobility species A \\
$M_{B}=10^{-17} \mathrm{~m}^{2} \mathrm{~s}^{-1}$ & mobility species B \\
$\bar{\gamma}_{A}=1$ & activity coefficient species A \\
$\bar{\gamma}_{B}=1$ & activity coefficient species B \\
$c_{A_{\max }}=1$ & total number of $\alpha$-lattice sites \\
$c_{B_{\max }}=1$ & total number of $\beta$-lattice sites \\
$\nu=0.22$ & Poisson's ratio \\
$\beta_{A}=\beta_{B}=0.0$ & relative lattice constants for the baseline case \\
$\beta_{A}=0.1 ; \beta_{B}=0.0$ & relative lattice constants rate for the Ab case \\
$E=100 \mathrm{GPa}$ & Young's modulus \\
\hline
\end{tabular}

The orange lines that are superimposed in the rightmost graph of Fig. $2 \mathrm{~b}$ result from a simulation that includes the stress dependent term of the diffusion potential in Eq. (48) by associating a $10 \%$ isotropic expansion to the intercalation of species $A^{+}$(Ab case). The Vegard strain is described by the anelastic deformation of Eq. 15. with $\beta_{A}$ being a constant value set to 0.1 , while $\beta_{B}$ is zero (Tab.1). Young's modulus and the Poisson's ratio are taken to have the constant values of $\mathrm{E}=50 \mathrm{GPa}, \nu=0.22$. The film develops a compressive stress as

365 high as of $1 \mathrm{GPa}$, but produces a negligible deviation of the concentration profile of both $A^{+}$and $B^{-}$.

The electroneutrality condition imposes a local constraint on the fluxes and therefore on the relation among the rates of change of species concentration

$$
\sum_{i} z_{i} \mathbf{J}_{i}=\mathbf{0} \Rightarrow \sum_{i} z_{i} \nabla \cdot \mathbf{J}_{i}=0 \Rightarrow \sum_{i} z_{i} \frac{\partial c_{i}}{\partial t}=0
$$

Note that $\sum_{i} z_{i} \partial c_{i} / \partial t=0$ implies that $\sum_{i} z_{i} c_{i}=$ constant. This constant is typically zero because the initial stoichiometry is charge neutral; therefore $\sum_{i} z_{i} c_{i}=0$. This is different from the steady state condition, which would read ${ }_{370} J_{i}=0$ for all $i$.

\subsection{Kinetic demixing}

In compounds containing multiple cations and anions, differences in the transport rates can lead to compositional separation during ambipolar diffusion. Kinetic demixing is an example of this phenomenon (distinguished from thermodynamic demixing such as spinodal decomposition) in which deviations from homogeneous compositions develop in ionic compounds 14. We will treat the case where demixing is caused by electro-chemo-mechanical potential gradients of oxygen such as in SOFCs. Kinetic demixing can couple to thermodynamic demixing (e.g. spinodal decomposition) though gradients of the diffusion potential.

In literature a classification of kinetic demixing (or decomposition) phenomena based on the type of driving force has been proposed [23. However, in most systems multiple effects and physics interact, so that this sort of distinction may 

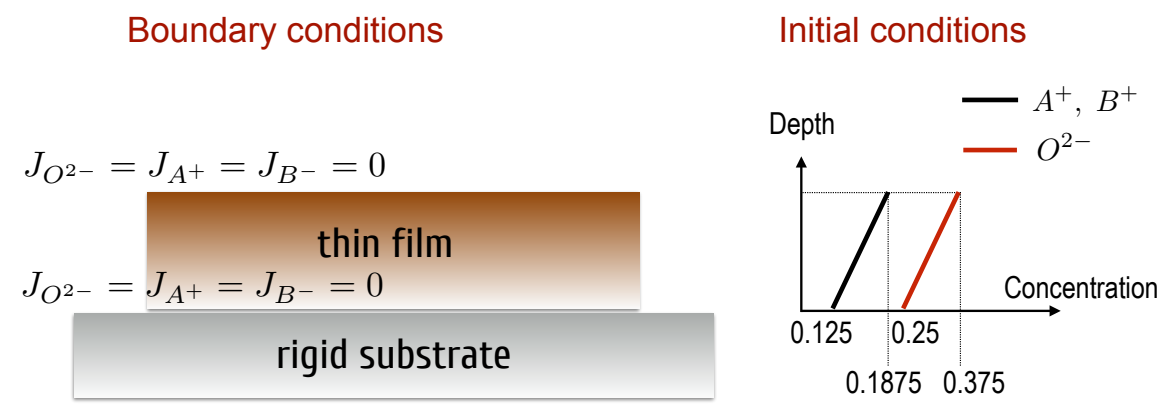

Figure 3: Schematic representation of the set up for the numerical tests presented in 4.2 .

become arbitrary and not very representative. We prefer to classify multifunctional oxide materials, and the eventual demixing (or decomposition) that they develop, according to the following categories:

- oxygen-preferred carriers, $M_{O^{-2}}>>M_{\text {cations }}$

- cation-preferred carriers, $M_{O^{-2}}<<M_{\text {cations }}$

Consider the experimental setup illustrated in Fig. 3. Geometry, mechanical 390 boundary conditions and discretization are the same as in Section 4.1.1. The thin film is a multicomponent oxide $\mathrm{A}_{1-x} \mathrm{~B}_{x} \mathrm{O}$, where $\mathrm{A}$ and $\mathrm{B}$ are cations, each with a lower mobility than oxygen's (this is typically the case for oxides used as SOFC cathode materials).

An initial oxygen potential gradient is created by imposing the $O^{2-}$ concentration to vary linearly over the film thickness at the beginning of the simulation

$$
c_{O^{2-}}(\xi, \tau=0)=m+n \xi ; \quad m=0.25 ; n=m / 2
$$

(as illustrated in Fig, 3). This approximates the condition of an SOFC cathode with different oxygen pressures acting on the two sides. The initial concentration of cations $\mathrm{A}^{+2}$ and $\mathrm{B}^{+2}$ is chosen to guarantee that stoichiometry and charge are balanced

$$
c_{A^{2+}}(\xi, \tau=0)=c_{B^{2+}}^{0}(\xi, \tau=0)=\frac{1}{2}(m+n \xi)
$$

By hypothesis, the total number of $\mathrm{A}$ sites (i.e., $\alpha$-lattice), $\mathrm{B}$ sites and $\mathrm{O}$ sites is conserved and, for each species, only one oxidation state is allowed.

The defect reaction, in the Kröger-Vink notation [36] is written as $\mathrm{A}_{(1-\mathrm{x})} \mathrm{B}_{\mathrm{x}} \mathrm{O}=(1-\mathrm{x}) \mathrm{A}_{\mathrm{A}}^{\times}+\mathrm{xB}_{\mathrm{B}}^{\times}+\mathrm{O}_{\mathrm{O}}^{\times}$ null $\longrightarrow(1-\mathrm{x}) \mathrm{V}_{\mathrm{A}}^{\prime \prime}+\mathrm{xV}_{\mathrm{B}}^{\prime \prime}+\mathrm{V}_{\mathrm{B}}^{\bullet \bullet}$ where $\mathrm{A}_{\mathrm{A}}^{\times}, \mathrm{B}_{\mathrm{B}}^{\times}$, and $\mathrm{O}_{\mathrm{O}}^{\times}$are cations and oxygen at their (mutually exclusive) lattice sites, $\mathrm{V}_{\mathrm{A}}^{\prime \prime}, \mathrm{V}_{\mathrm{B}}^{\prime \prime}$, and $\mathrm{V}_{\mathrm{O}}^{\bullet \bullet}$ are the respective charged vacancies. The network 
constraint on the total number of lattice sites available for ions and vacancies is

$$
\begin{aligned}
& c_{\mathrm{A}_{\mathrm{A}}^{\times}}+c_{\mathrm{V}_{\mathrm{A}}^{\prime \prime}}=c_{A_{\max }}=\text { constant } \\
& c_{\mathrm{B}_{\mathrm{B}}^{\times}}+c_{\mathrm{V}_{\mathrm{B}}^{\prime \prime}}=c_{B_{\max }}=\text { constant } \\
& c_{\mathrm{O}_{\mathrm{O}}^{\times}}+c_{\mathrm{V}_{\mathrm{O}}^{\bullet \bullet}}=c_{O_{\max }}=\text { constant }
\end{aligned}
$$

and the corresponding electrochemical potentials take the form

$$
\begin{aligned}
& \mu_{A}^{E C}\left(c_{A}\right)=R T \ln \frac{c_{A}}{c_{A_{\max }}-c_{A}}+R T \ln \bar{\gamma}_{A} \\
& \mu_{B}^{E C}\left(c_{B}\right)=R T \ln \frac{c_{B}}{c_{B_{\max }}-c_{B}}+R T \ln \bar{\gamma}_{B} \\
& \mu_{O}^{E C}\left(c_{O}\right)=R T \ln \frac{c_{O}}{c_{O_{\max }}-c_{O}}+R T \ln \bar{\gamma}_{O}
\end{aligned}
$$

Table 2: Material parameters for problems in Section 4.2

\begin{tabular}{ll} 
Input value & Description \\
\hline$F=96485.3365 \mathrm{C} \mathrm{mol}^{-1}$ & Faraday's constant \\
$R=8.314 \mathrm{~J} \mathrm{~K}^{-1} \mathrm{~mol}^{-1}$ & gas constant \\
$T=298 \mathrm{~K}$ & Temperature \\
$M_{O}=10^{-13} \mathrm{~m}^{2} \mathrm{~s}^{-1}$ & oxygen mobility \\
$M_{A}=10^{-16} \mathrm{~m}^{2} \mathrm{~s}^{-1}$ & mobility species A \\
$M_{B}=10^{-18} \mathrm{~m}^{2} \mathrm{~s}^{-1}$ & mobility species B \\
$\bar{\gamma}_{O}=1$ & oxygen activity coefficient \\
$\bar{\gamma}_{A}=1$ & activity coefficient species A \\
$\bar{\gamma}_{B}=1$ & activity coefficient species B \\
$c_{O \max }=1$ & total number of oxygen lattice sites \\
$c_{A_{\max }}=1$ & total number of $\alpha$-lattice sites \\
$c_{B \max }=1$ & total number of $\beta$-lattice sites \\
$\nu=0.22$ & Poisson's ratio \\
$\beta_{O}=\beta_{A}=\beta_{B}=0$ & relative lattice constants for the baseline \\
$\beta_{O}=\beta_{B}=0 ; \beta_{A}=0.2$ & case \\
$\beta_{O}=\beta_{A}=0 ; \beta_{B}=0.2$ & relative lattice constants for the Ab case \\
$E=100 \mathrm{GPa}$ & relative lattice constants for the Ba case \\
\hline
\end{tabular}

To isolate the transient effects of an electro-chemo-mechanical potential gradient, zero flux boundary condition are applied at the two surfaces. Electrostatic forces strongly limit the local charge imbalances; nevertheless, kinetic demixing (i.e., stoichiometric departures from the equilibrium state) appears in the simulation. We will assume the solution to be ideal, i.e., $\bar{\gamma}_{O}=\bar{\gamma}_{A}=\bar{\gamma}_{B}=1$.

The three mobile species allow kinetic demixing. The electroneutrality condition (Eq. (52) ) and the stoichiometry constraint $\left(\sum_{i} z_{i} c_{i}=0\right)$ imply that the concentration of one species is a linear combination (with coefficients $z_{i}$ ) of the other two. The extra degree of freedom allows the concentration ratio of $A^{2+}$ to $B^{2+}$ to vary (i.e., $x$ in $\mathrm{A}_{1-x} \mathrm{~B}_{x} \mathrm{O}$ is a function of position and time) and therefore demixing is possible.

${ }_{410}$ In perovskite oxides, A-site cations usually exhibit higher diffusivity than Bsite cations, and for SOFC cathodes oxygen has the highest diffusivity. In this simulation, we have assumed $M_{O^{-2}}=10^{3} \times M_{A^{+2}}=10^{5} \times M_{B^{+2}}$. The results 


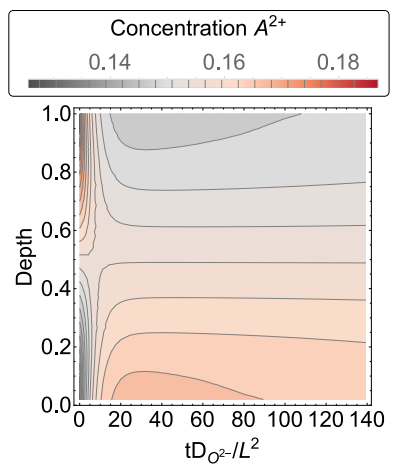

(a)

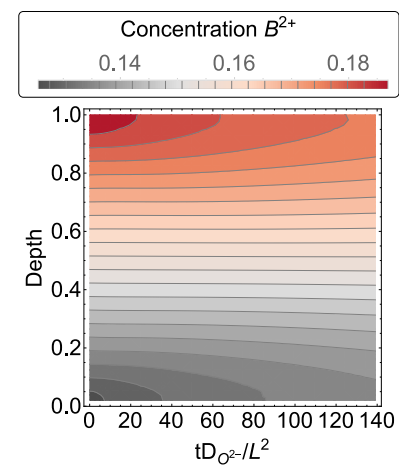

(b)

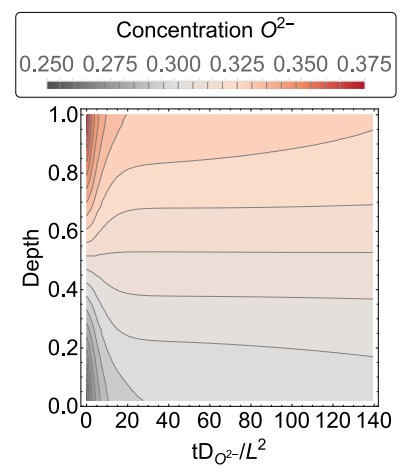

(c)

Figure 4: The time-dependent concentration fields for the three species forming an oxide $\mathrm{A}_{1-x} \mathrm{~B}_{x} \mathrm{O}$.

are presented as non-dimensionalized as in Sec. 4.1.1.

Fig. 4 shows how each concentration field evolves over the first $5 \%$ of the simulation time. The oxygen potential gradient drives the diffusion of $\mathrm{O}^{-2}$ ions (Fig. 4c) which are coupled with the transport of cations because of charge neutrality. Since $\mathrm{A}^{2+}$ is the faster of the two cation species, it can couple to the $\mathrm{O}^{-2}$ flux to maintain charge balance: the transport of oxygen is limited by the faster cations (Fig. 4a). The $\mathrm{B}^{2+}$ redistribution occurs over a much longer time 420 scale; its diffusion potential is reduced (Fig. 4b).

Let us focus on what happens at the two surfaces of the sample. Changes in surface chemistry are relevant to the behavior of SOFC cathodes. To simulate the combined effect of induced Vegard's stress and displacement boundary conditions, we performed two simulations: 1) a Vegard's expansion is assigned to the higher mobility cation only; 2) the expansion is assigned to the lower mobility cation. The transient segregation is characterized by the time-dependent values at the top (stress free) and bottom (fixed grip) surfaces.

Fig. 5 a collects the data for a baseline case where both A or B have zero Vegard's coefficients. At the top surface the concentration of cation $\mathrm{A}^{2+}$ undergoes

430 a sudden drop followed by a gradual increase, while the concentration of cation $\mathrm{B}^{2+}$ monotonically decreases. The reversed situation is predicted at the bottom surface. Cations segregation is present during the transient state, while homogeneity is restored when the system approaches its steady state. This is also clearly represented in Fig. 6a, where the local stoichiometric imbalance of cations is plotted over time across the entire spatial dimension.

Conversely, demixing develops and is maintained at the steady state in the two following cases

Ab : cation $A^{2+}$ characterized by $20 \%$ of isotropic lattice mismatch and none for $B^{2+}$. 

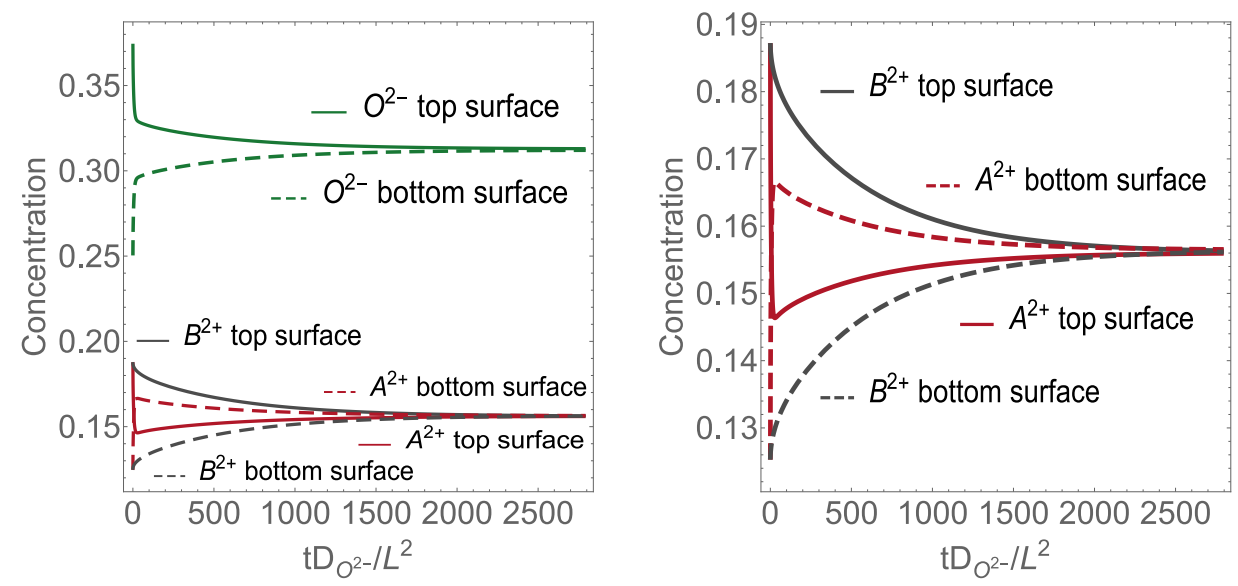

(a) Concentration of cations and oxygen at the top and bottom surface of the unstressed film. The analysis predicts cations demixing during the transient state, and homogeneous composition once the steady state is reached.
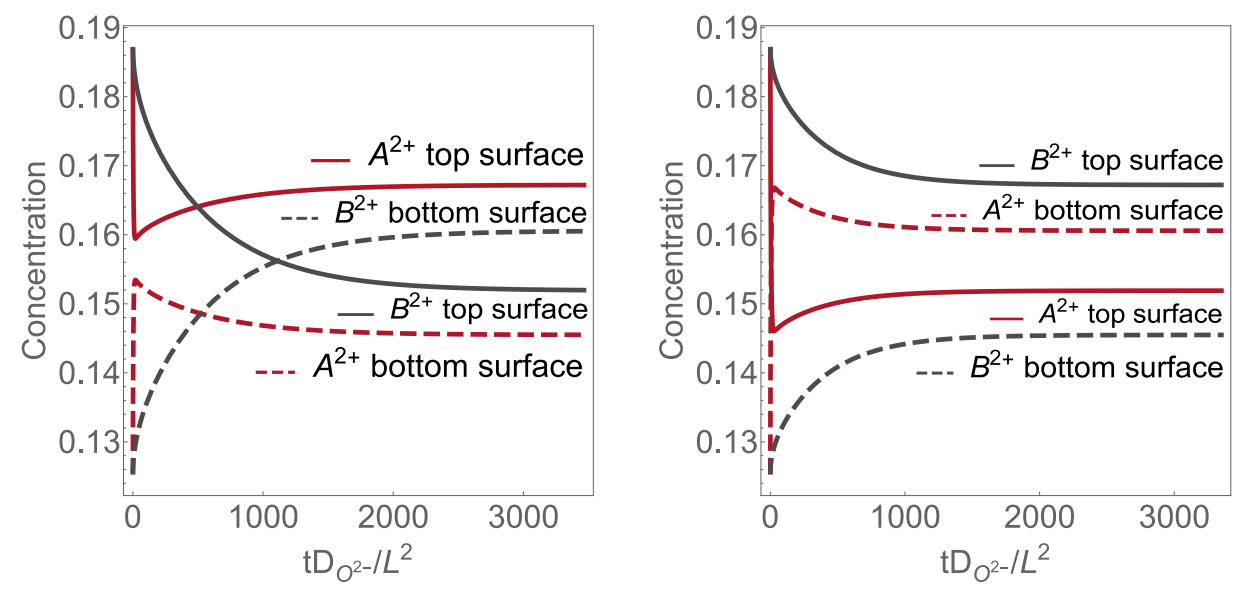

(b) Evolution of the cation concentrations at the top (free boundary) and bottom (fixed grip) interfaces of the film for two cases of assumed Vegard's stresses (left: $20 \%$ of lattice mismatch for species A with respect to host; right: $20 \%$ of relative lattice mismatch for species B). In both cases, $M_{O^{-2}}=10^{2} \times M_{A^{+2}}=10^{4} \times M_{B^{+2}}$. Both simulations predict demixing: in the first case $\mathrm{A}^{2}+$ segregates to the top and $\mathrm{B}^{2}+$ segregates to the bottom surface. The segregation behavior reverses for the second choice of Vegard coefficients.

Figure 5: Example of kinetic demixing promoted by stress potential gradient. 
Fig. 7 focuses the attention on the maximum stoichiometric imbalance reached across the film thickness over time. Fig. $7 \mathrm{~b}$ shows that the maximum amount of demixing within the sample is the same for $\mathbf{A b}$ and $\mathbf{B a}$ even though they have different time-dependent concentration fields and different assumption on the 


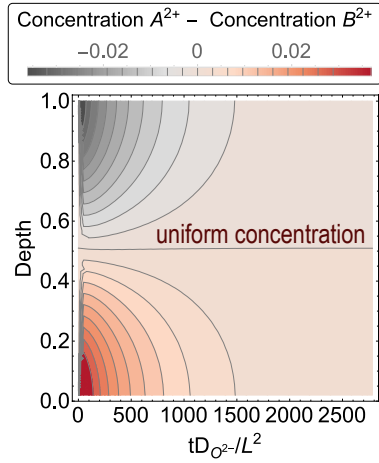

(a)

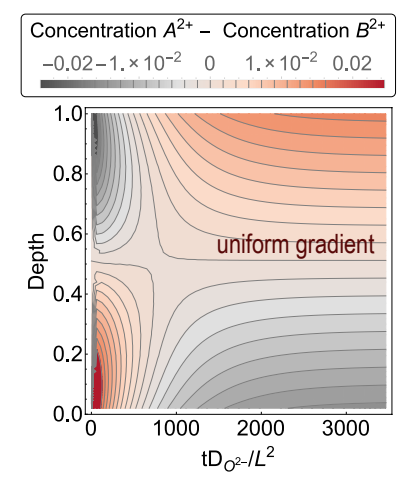

(b)

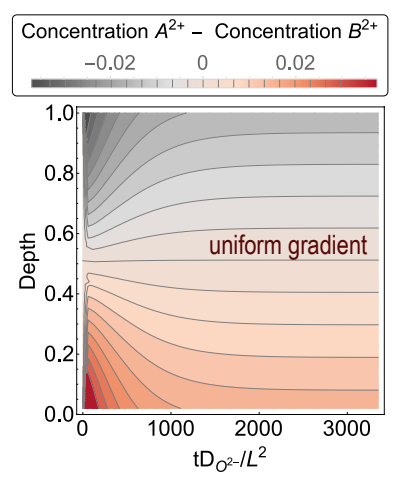

(c)

Figure 6: Local stoichiometric imbalance between cation $\mathrm{A}^{2+}$ and cation $\mathrm{B}^{2+}$. The left figure refers to the baseline (no Vegard's stresses) case and it shows no cations segregation at the steady state. Fig. 6b (Ab case) exhibits, at the steady state, a higher concentration of $\mathrm{A}^{2+}$ at the top surface and localization of $\mathrm{B}^{2+}$ at the bottom one. This condition is achieved after an initial concentration polarization of opposite sign. In Fig. 6c (Ba case) the system monotonically approaches a non-homogeneous condition with $\mathrm{B}^{2+}$ segregated at the top and $\mathrm{A}^{2+}$ at the bottom surface.

atom sizes. The understanding of how Vegard's stresses and mobility produce different types of demixing provides materials-design criteria for SOFC cathode materials.

Further numerical tests have demonstrated that the amount of cations stoichiometric imbalance arising in oxides depends on both the initial oxygen potential gradient and on the intensity of the stress field induced by chemical swelling/deswelling of the lattice.

For demixing to occur, it is necessary that the cations have different diffusivities. However, because diffusivity depends on mobility and concentration $(D=c M)$, it is coupled to stress in two possible ways: through the dependence of concentration on the local stress or by a stress-dependent mobility. In the simulations presented herein, the mobility is assumed to be constant. Therefore, due to the effect of Vegard's stresses, the conditions for demixing can appear for cations with similar mobility.

The magnitude of demixing does not depend on differences of the $\mathrm{A}^{2+}$ and $\mathrm{B}^{2+}$

500 diffusivities: there is a critical difference at which demixing appears. Once this critical value is attained, further increases only reduce the time required for the system to reach the steady state, but it does not produce enhanced demixing.

\subsection{Mechanically driven diffusion}

Finally, a three dimensional finite element simulation of a mechanically loaded crack in a lithium-intercalating electrode. Cracking is often observed in the 


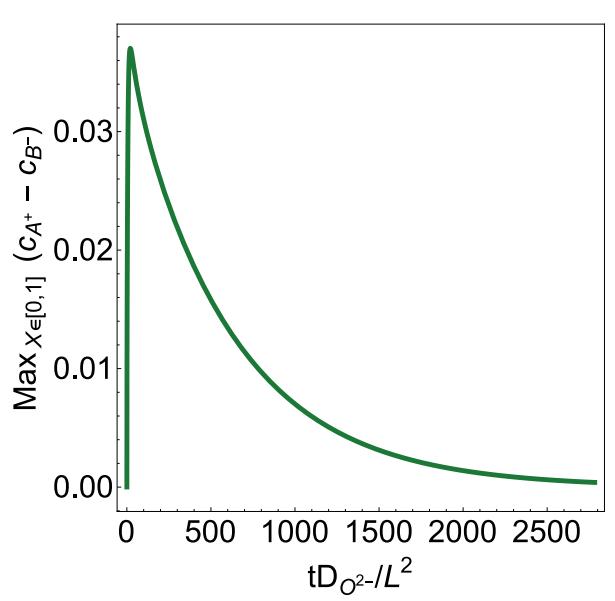

(a)

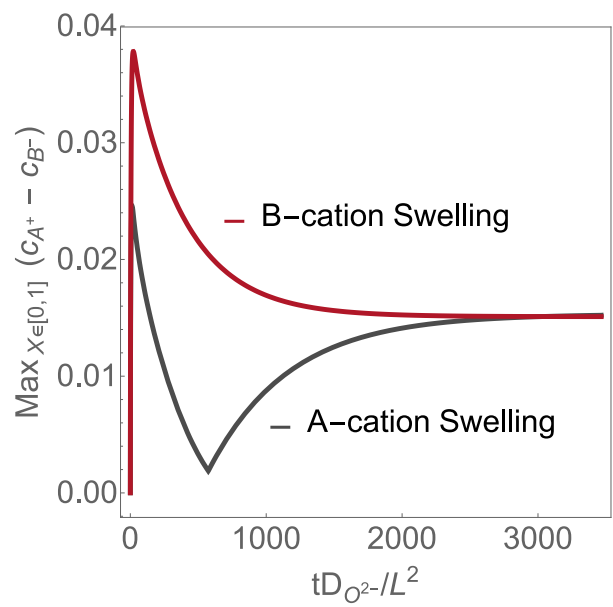

(b)

Figure 7: Representation of the maximum stoichiometric imbalance reached in favor of the A-cation. The imbalance converges to zero when no mechanical stress is acting on the diffusion.

active material of composite electrodes of Li-ion batteries. The presence of fracture in the electrode particles perturbs the stress field and results in stress concentration around the crack tips, which locally affects lithium concentration. This may prevent full lithiation and promote further fracture propagation.

510 A simulation of a notched sample under tensile loading is used to investigate the effect of stress gradient on diffusion. The sample geometry is chosen to satisfy the ASTM standard test for fracture toughness measurements. A farfield tensile stress of $10 \mathrm{MPa}$ is applied in the direction normal to the crack faces, giving a Mode-I fracture conditions. The material parameters selected

515 for this problem are listed in Table 3 . Governing and constitutive equations are summarized in Appendix B.

From the initial uniform concentration, we calculate the redistribution of $\mathrm{Li}$ atoms within the sample under the sole effect of mechanical loading. Ideal solution behavior, and fully nonlinear kinematic treatment are assumed. Zero 20 external Li flux boundary conditions are applied. The change in concentration arises only due to the nonuniform stress field, and in particular to the localization of stress ahead of the crack tip. The development of an area of high tensile stress and of high stress gradient produces a driving force for the diffusion of lithium.

${ }_{525}$ A systematic study of Li redistribution in the presence of cracks, and its role in fracture propagation has been carried out for elastic and plastic material [37. Such study considers the system at its steady state, and looks at results attained for an increasing far field load. On the contrary, we hold the external load fixed at $10 \mathrm{MPa}$ and follow the system evolution up to steady state. We are inter- 

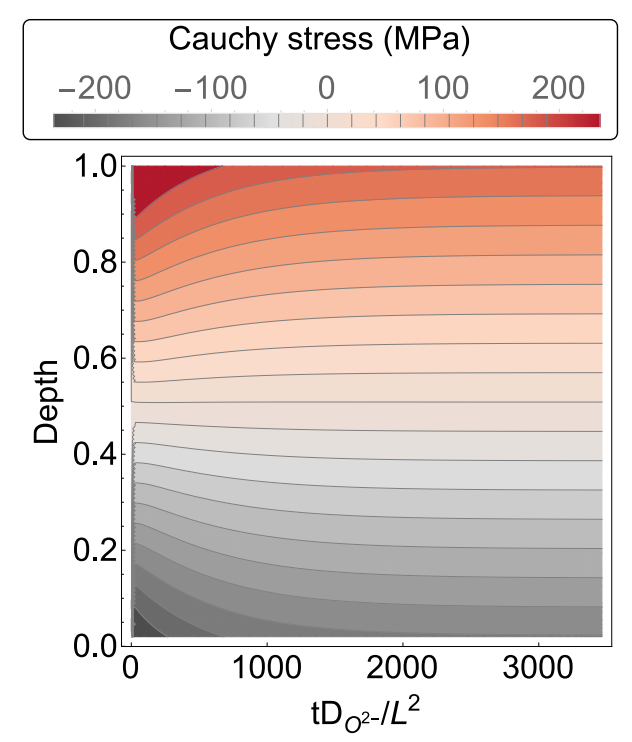

(a) Ab case (cation $\mathrm{A}^{2+}$ swelling)
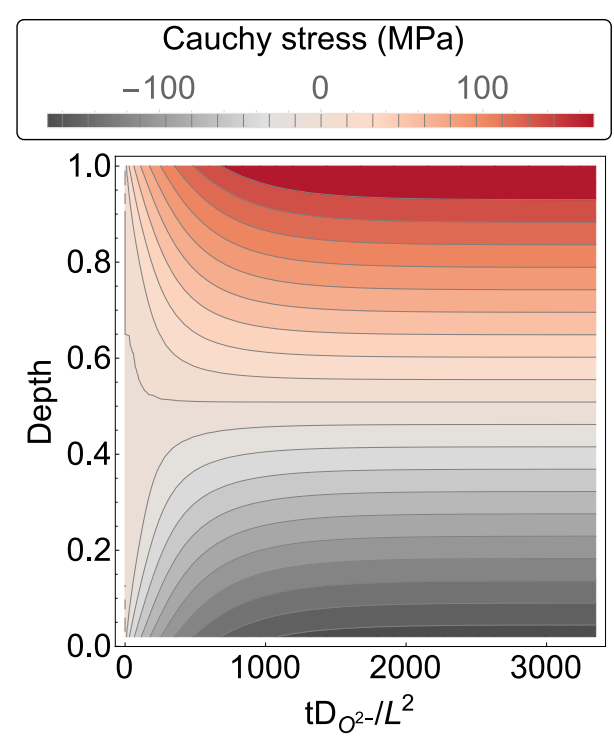

(b) Ba case (cation $\mathrm{B}^{2+}$ swelling)

\section{thin film free to deform}

\section{thin film constrained by the substrate}

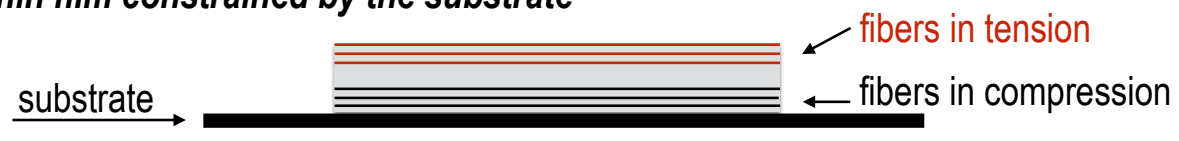

(c) Schematic representation of the volumetric deformation of opposite sign arising in the top and bottom half of the sample for both ( $\mathbf{A} \mathbf{b}$ and $\mathbf{B a}$ ) examples. The film is assumed to be fixed to a rigid substrate and characterized by a biaxial plane-stress state, arising from the fact the the film is not allowed to relax in the bent configuration showed above.

Figure 8: Stress field produced by the chemical expansion associated with the intercalation of species A (Ab case) or species B (Ba case). 

misfit parameter, both affecting the Vegard's stress and the chemo-mechanical coupling.

We assume a positive anelastic lattice deformation upon intercalation (see equation 17 with $\beta_{L i}>0$ ). Consequently $\mathrm{Li}$ is driven towards areas of higher tensile

535 stress ahead of the crack. The sign of the stress-dependent driving force for diffusion depends on the sign of $\beta_{L i}$, while its value is a function of the absolute value of $\beta_{L i}$ as well as on the elastic properties of the material (refer to Eq. 46) for the diffusion potential of neutral species).

If open-circuit potential versus concentration data are available, the term $R T \ln \bar{\gamma}$

540 would be included in the diffusion potential by following the procedure described in 38 .

The simulation conditions are illustrated in Fig. 9a.

A constant load of $10 \mathrm{MPa}$ was applied and the resulting fluxes were computed until the composition reached a steady-state distribution. The configuration in Fig. 9a was discretized with linear quadrilateral elements, and with a nonuniform refinement in the crack tip region. The use of non-conforming elements is handled via the deal.II finite element library [39]. Fig. 9a shows a contour plot of $\mathrm{Li}$ concentration and out of plane displacement field for the baseline case. In the image the sample's deformation is scaled by a factor of 50 in order to make

550 it clearly visible. An initial uniform concentration $\left(\zeta_{L i} \equiv c_{L i} / c_{L i_{\max }}=0.5\right)$ is assumed throughout the sample, and the material properties are $\mathrm{E}=75 \mathrm{GPa}$ and $\beta=0.085$. The contours represent the steady-state condition. Ahead of the crack-tip the relative concentration increases to $\zeta_{L i}=0.592$, a $18.4 \%$ relative enhancement. The time-dependence of this enhancement is shown in Fig. $9 \mathrm{~b}$

555 Multiple sets of results are presented in Fig. 9b and Fig. 9c Each test is characterized by a different value of Young's modulus or lattice misfit parameter with respect to the baseline example. An additional test was performed by adding a plain strain constraint to the reference case, with the aim of investigating the effect of a simplified two-dimensional treatment of the problem.

560 For the case of less stiff $(\mathrm{E}=15 \mathrm{GPa})$ material, the maximum concentration reached at the crack tip shows a reduced, but significant, $16.8 \%$ enhancement. Lower values of volumetric expansion of $5 \%$ and $15 \%$ produce respectively a $3 \%$ and $10 \% \mathrm{Li}$ redistribution. These results show a stronger dependence on the Vegard's lattice parameter, rather than on the Young's modulus.

565 The out of plane displacement field in 9a is caused by Li non-uniform distribution, since the Poisson's ratio is fixed to zero. By constraining the displacement in the $\mathrm{Z}$ direction, we recreate plain strain condition, one of the assumption in [37. As a consequence of the plain strain condition, a larger out of plane compressive stress develops upon Li redistribution around the crack tip. This, in

570 turn, inhibits further diffusion and a lower value of $\mathrm{Li}$ concentration is reached at the crack tip. The plot in Fig. $9 \mathrm{~b}$ shows how the concentration for this case attains values close to the example with $15 \%$ volumetric expansion.

The analyses were performed in finite strain, and the approximation of the stress in the area surrounding the crack tip is expected to improve upon mesh 575 refinement. However, it is beyond the scope of the present work to accurately 
predict the stress at the crack tip. We propose this example mainly to illustrate how mechanical stress can produce a non-negligible driving force for diffusion. The model is capable to capture Li redistribution under the sole effect of a stress gradient field. In in Fig. $9 \mathrm{~b}$ we intend to compare the time evolution of the hydrostatic stress at the crack tip for problems with different input parameters or boundary conditions. In particular we are interested in observing the effect of the Young modulus, Vegard's lattice parameter, and plane strain boundary conditions.

The initial relaxation of the hydrostatic stress observed in Fig. 9c is due to the development of a compressive out of plane stress, while the Cauchy stresses in the XY plane stay tensile and only moderately change over time. As expected, the largest out of plane stress arises in the constrained plane strain condition. The results for the test with lower Young's modulus (15 GPa v.s. $75 \mathrm{GPa}$ ) or lower anelastic deformation (5\% and $15 \%$ of swelling v.s. $28 \%$ ) are rather similar in value and they follow the trend of the baseline case, showing and initial drop followed by a gradual increase. At steady state the hydrostatic stress recovers approximately the value computed at the beginning of the simulation, before diffusion comes into play. The system requires a longer time to reach steady state when the material is stiffer. In [37] the authors predict a monotonically 595 decreasing hydrostatic stress, this is due to the imposed plain strain condition. The examples presented in this section are meant to to emphasize the role of stress as driving force in cases where intercalation is coupled with anelastic deformation of the hosting lattice. Even in presence of a moderate value of volumetric expansion rate, mechanical stress can be responsible for a driving force large enough to induce a considerable Li redistribution.

Table 3: Material parameters for problems in Section 4.3

\begin{tabular}{ll} 
Input value & Description \\
\hline$F=96485.3365 \mathrm{C} \mathrm{mol}^{-1}$ & Faraday's constant \\
$R=8.314 \mathrm{~J} \mathrm{~K}^{-1} \mathrm{~mol}^{-1}$ & gas constant \\
$T=298 \mathrm{~K}$ & temperature \\
$M_{L i}=0.60 \cdot 10^{-12} \mathrm{~m}^{2} \mathrm{~s}^{-1}$ & mobility \\
$\gamma_{L i}=1$ & activity coefficient \\
$\zeta_{L i}=0.76$ & relative initial concentration \\
$\nu=0$ & Poisson's ratio \\
$\beta_{L i}=0.085$ & relative lattice constant for the baseline case $(28 \%$ swelling $)$ \\
$\beta_{L i}=0.0164$ & relative lattice constant for case with $5 \%$ swelling \\
$\beta_{L i}=0.0477$ & relative lattice constant for case with $15 \%$ swelling \\
$E=75 \mathrm{GPa}$ & Young's modulus for the baseline case \\
$L=0.625 \cdot 10^{-3} \mathrm{~m}$ & half of the sample edge length \\
\hline
\end{tabular}

\section{Conclusions}

We have developed a continuum-level theory which couples the transport of multiple charged species with the deformation of the solid medium. It includes a fully nonlinear kinematic description of the anelastic volumetric expansion of 605 the hosting lattice, caused by the intercalating particles with lattice network 


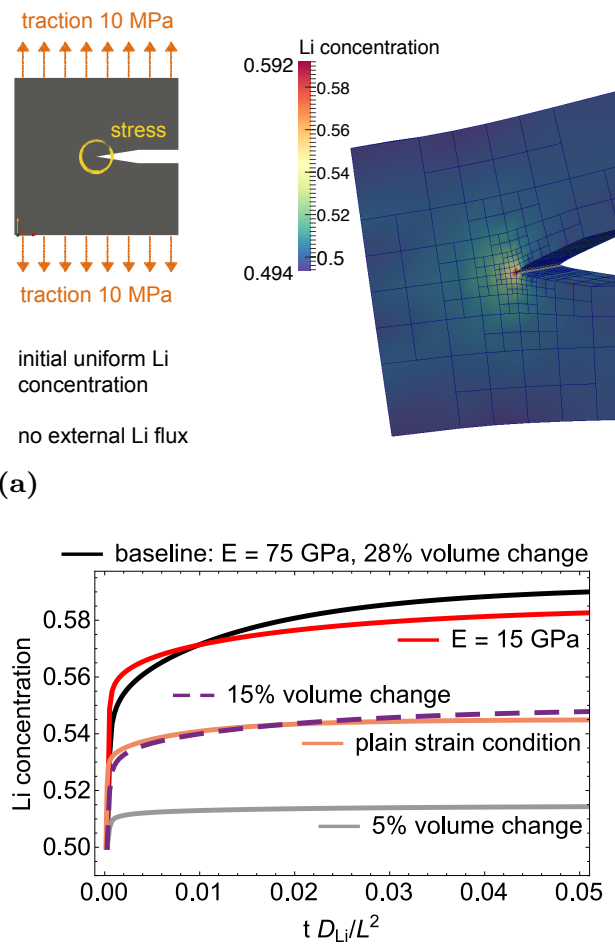

(b)

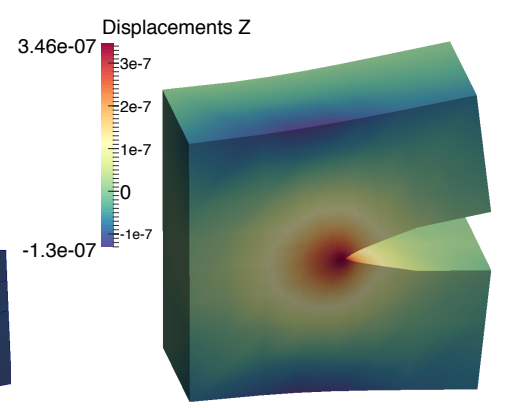

Figure 9: Simulation of a traction load applied to a notched sample. From the initial uniform concentration $\left(\zeta_{L i} \equiv c_{L i} / c_{L i_{\max }}=0.5\right)$, the 3D contour plot in $9 \mathrm{a}$ shows the steady state distribution of Li for a material with Young's modulus $\mathrm{E}=75 \mathrm{GPa}$. Having assumed Poisson's ratio equal to zero, the out of plane displacement field shown in 9a arises from Li non-uniform distribution. Zero external Li flux is applied, and the change in concentration arises only due to the nonuniform stress field, and in particular to the localization of stress ahead of the crack tip. The development of an area of high tensile stress and of high stress gradient produces a driving force for the diffusion of lithium. Graphs in Fig. 9b shows how Li concentration evolves over time at the crack tip. For the case of $\mathrm{E}=15 \mathrm{GPa}$, the maximum concentration reached at the crack tip is equal to $\zeta_{L i}=0.584,16.8 \%$ higher than the initial value. In the analysis with $\mathrm{E}$ $=75 \mathrm{GPa}$ the relative concentration grows up to $\zeta_{L i}=0.592$, a $18.4 \%$ increment respect to the value away form the crack. If a plain strain boundary condition is imposed, out of plane expansion is prevented and Li concentration only raise up to $9 \%$. Fig. $9 \mathrm{~b}$ compares results from analysis characterized by various volumetric expansion rates, thus showing how the lattice mismatch parameter largely affects the chemo-mechanical coupling. Fig. 9c shows the time evolution of the hydrostatic stress at the crack tip. The development of compressive out of plane stress is responsible for the initial hydrostatic stress relaxation. If out of plane deformation is allowed, the hydrostatic stress tends to grow back to its initial value. 
constraints. Electrostatic forces couple the diffusion of ions, as an internal electric field arises to counteract long range charge separation. The system kinetics are driven by the concurrent action of electro-chemo mechanical forces and are able to simulate the behavior of multifunctional materials.

The purpose of this development is the study of the technologically relevant cases of diffusion of ions in electrode and solid electrolyte materials for lithiumion batteries and solid oxide fuel cells. The theory, its reduction to a finite element formulation, and the numerical methods for its solution are targeted to perform solutions on the complicated microstructures that occur in batteries ${ }_{615}$ and SOFCs. In this paper, we demonstrate that the method reproduces sensible physics in two simple cases. However, these simple cases do shed light on relevant phenomena.

In the case of two-species ambipolar transport, where diffusion takes place by vacancies produced by Schottky defects, no charge imbalance or kinetic demixing can occur except for extraordinarily high stresses or chemical driving forces. This results from the relative magnitude of the electrostatic energy, with respect to the mechanical and chemical energy stored.

Conversely, kinetic demixing is observed in the diffusion of three ionic species within an oxide material. The combined effect of Vegard's stresses and different ${ }_{625}$ cations diffusivity, result in a non-homogeneous electrode composition. In the absence of mechanical coupling, the transient demixing vanishes as the system approaches its steady state. The results presented in 4.2 emphasize the relevance of mechanical coupling in the treatment of defect-migration problems. The proposed examples show how the fully coupled model is an appropriate tool to ${ }_{630}$ explain the formation of a cation rich surface layer in SOFC cathodes. This phenomenon is detrimental to the cathode oxygen-reduction catalytic-efficiency, and it is considered to be the main barrier for implementation of high-performance SOFCs at intermediate temperatures $500-700^{\circ} \mathrm{C}$.

To study diffusion under the effect of a highly non-homogeneous stress field, 635 in 4.3 we proposed A 3D simulation of a notched sample under tensile loading. A significant Li redistribution around the crack tip is observed in consequence of stress localization.

Simulations of mechanically loaded cracks in electrodes indicate that significant intercalated Li concentration gradients can develop in the vicinity of the crack ${ }_{640}$ tip. Such inhomogeneous concentrations will affect fluxes from the electrolyte into the electrode. Furthermore, cyclic loading would incur energetic losses due to the redistribution of $\mathrm{Li}$ at each cycle.

The theory and its numerical implementation are quite general, and their application is not limited to the examples illustrated herein. A variety of electrochemical645 mechanical systems and materials can be modeled at the mesoscopic scale; rigorous investigations can be carried out to identify the underlying circumstances which compromise their life and performance. 


\section{Acknowledgments}

The authors are very grateful to Prof. Sean R. Bishop, Prof. Brian Sheldon, ${ }_{650}$ Prof. Harry L. Tuller, and Prof. Bilge Yildiz for their valuable comments, and for drawing important references to our attention. The work was supported by the grant DE-SC0002633 funded by the U.S. Department of Energy, Office of Science. We are appreciative of the meticulous work of an anonymous reviewer. His comments helped us considerably improve the manuscript, by making the message more clear and the content more accessible.

\section{Appendix A. Nomenclature}

Table A.4: Glossary of symbols

\begin{tabular}{|c|c|}
\hline Symbol & Description \\
\hline B & body force per unit of reference volume \\
\hline $\mathbf{C}^{e}$ & elastic right Cauchy-Green strain tensor \\
\hline $\mathbb{C}$ & elasticity tensor \\
\hline$D_{i}$ & diffusivity of species $i$ \\
\hline $\mathcal{D}$ & dissipation density \\
\hline $\mathbf{E}$ & electric field in the reference configuration \\
\hline$F$ & Faraday's constant \\
\hline $\mathcal{F}^{e x t}$ & vector of external forces in the finite element discretization \\
\hline $\mathcal{F}^{i n t}$ & vector of internal forces in the finite element discretization \\
\hline $\mathbf{F}$ & deformation gradient \\
\hline $\mathbf{F}^{e}$ & elastic deformation gradient \\
\hline $\mathbf{F}^{a}$ & anelastic deformation gradient \\
\hline$G$ & shear modulus or second Lamé constant \\
\hline$I_{1}$ & first invariant of the elastic right Cauchy-Green strain tensor $I_{1}=\operatorname{tr}\left(\mathbf{C}^{\mathbf{e}}\right)$ \\
\hline$J$ & Jacobian, $J=\operatorname{det}\left(\mathbf{F}^{e}\right)$ \\
\hline $\bar{J}$ & imposed normal flux on the Neumann boundary \\
\hline $\mathbf{J}_{i}$ & outward flux of species $i$, measured per unit of undeformed area per unit time \\
\hline $\mathbf{J}_{Q}$ & outward heat flux, measured per unit of undeformed area per unit time \\
\hline $\mathbf{J}_{q_{i}}$ & outward flux of charge $i$ per unit of undeformed area per unit time \\
\hline $\mathcal{K}^{1 / 2}$ & stiffness matrix in the finite element discretization \\
\hline$L$ & film thickness \\
\hline$M_{i}$ & mobility of species $i$ \\
\hline$N$ & finite element shape function \\
\hline $\mathcal{M}$ & mass matrix in the finite element discretization \\
\hline $\mathbf{P}$ & first Piola-Kirchhoff stress tensor \\
\hline $\mathbf{P}^{e}$ & equilibrium first Piola-Kirchhoff stress tensor \\
\hline$Q$ & distributed heat source per unit of reference volume \\
\hline$R$ & gas constant \\
\hline $\mathcal{R}$ & vector of residuals in the finite element discretization \\
\hline$T$ & absolute temperature \\
\hline$\overline{\mathbf{T}}$ & applied traction \\
\hline$U$ & internal energy per unit of undeformed volume $V_{0}$ \\
\hline$c_{i}$ & number of moles of the chemical species $i$ per unit reference volume $V_{0}$ \\
\hline$c_{i_{\max }}$ & total number of sites per unit reference volume $V_{0}$ available to the species $i$ \\
\hline$c_{0}$ & initial concentration \\
\hline $\bar{c}$ & imposed concentration on the Dirichlet boundary \\
\hline$c_{0}$ & initial concentration \\
\hline e & electric field in the spatial configuration \\
\hline$n_{i}$ & number of moles of the chemical species $i$ \\
\hline$q_{i}$ & charge of the chemical species $i$ per unit volume \\
\hline$t$ & time \\
\hline $\mathbf{u}$ & displacement \\
\hline$v$ & finite element test function \\
\hline$W_{\text {ext }}$ & external power \\
\hline$z_{i}$ & valence of species $i$ \\
\hline$\Upsilon^{M}$ & elastic free energy density \\
\hline
\end{tabular}


Table A.4: Glossary of symbols

\begin{tabular}{ll} 
Symbol & Description \\
\hline$\Upsilon^{E C}$ & electrochemical free energy density \\
$\Upsilon_{I I}^{E C}$ & excess of electrochemical free energy density for non-ideal solution \\
$\alpha$ & parameter in the trapezoidal time stepping algorithm \\
$\beta_{i}$ & relative lattice constant of species i intercalating into hosting material \\
$\gamma$ & activity coefficient \\
$s$ & entropy per unit of reference volume \\
$\lambda$ & first Lamé constant \\
$\mu^{\Theta}$ & chemical potential at the system reference state \\
$\mu_{i}^{E C}$ & electrochemical potential of the $i-t h$ chemical species \\
$\mu_{i}^{M}$ & stress dependent component of the diffusion potential of species $i$ \\
$\nu$ & Poisson's ratio \\
$\varpi_{i}$ & diffusion potential of the $i-t h$ chemical species \\
$\rho_{h}$ & molar density of the hosting compound \\
$\phi$ & electropotential \\
$\chi$ & deformation mapping \\
$\bar{\chi}$ & imposed value of the deformation mapping on the Dirichlet boundary \\
\hline
\end{tabular}

\section{Appendix B. Incremental boundary value problem in discrete form.}

The system presented herein is governed by two equations: the linear momentum balance and the mass balance, having displacements and concentration as 660 unknowns.

In the standard formulation, the diffusion potential $\varpi$ can be computed from the concentration and the stress field, which in turn is recovered from the displacements by Eq. 433 or some other similar law. Their computation requires the derivatives of the displacement field $u$. Furthermore the increment of concen665 tration $\dot{c}$ is function of the gradient of the diffusion potential. From a numerical point of view, differentiating implies a loss of precision. It is therefore appealing to look for a formulation in which constraints are readily accessible.

Given the physical 'importance' of $\varpi$, whose gradient appears as the driving force for diffusion, we choose to adopt a mixed finite element method [26] by 670 introducing the stress dependent component of the diffusion potential $\mu^{M}$ as a third independent variable.

In [40] only the displacements and the chemical potentials are taken as unknowns. This is option implies a few disadvantages, and it is possible only in cases where the diffusion potential does not account for the electrostatic driving force.

\section{Appendix B.1. Governing equations}

We introduce the static and transport problem, with their relative state functions, boundary and initial conditions. A numerical approximation of the system governing equations (written, for simplicity, by considering only one diffusing species) is constructed according to the finite element theory. Readers interested in the complete derivation can refer to the online supporting content. 
Appendix B.1.1. Static problem

The linear momentum balance in strong form can be written as

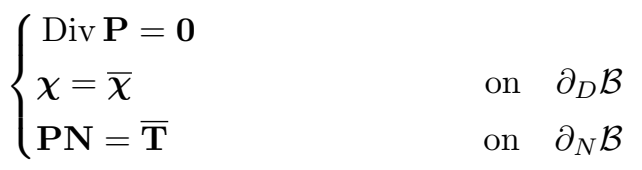

with Neumann and Dirichlet boundary conditions imposed on subsets of the domain boundary.

The discrete version of the static problem in the unknowns $\mathbf{u}$ and $\Delta \mathbf{c}$ can be written as 18

$$
\mathcal{K}^{u u} \mathbf{u}+\mathcal{K}^{u c} \Delta \mathbf{c}=\mathcal{R}^{u}
$$

where the residual $\mathcal{R}^{u}$ is computed as

$$
\mathcal{R}^{u}=\mathcal{F}_{\chi}^{e x t}\left(t_{n+1}\right)-\left.\mathcal{F}_{\chi}^{i n t(k)}\right|_{n+1}
$$

and the blocks of stiffness matrix are (with indices $A=1, \ldots N, B=1, \ldots N$ )

$$
\begin{aligned}
\mathcal{K}^{u u} & =\int_{\Omega_{e}} \nabla N^{b}: \mathbb{C}: \nabla N^{a} d V \\
\mathcal{K}^{u c} & =\int_{\Omega_{e}}\left(\frac{\partial \mathbf{P}}{\partial c} N^{b}\right): \nabla N^{a} d V
\end{aligned}
$$

with the following definitions of internal and external forces

$$
\begin{aligned}
& \mathcal{F}_{\chi}^{\text {int }}(\chi(t))=\int_{\mathcal{B}} \mathbf{P} \cdot \nabla N^{a} d V \\
& \mathcal{F}_{\chi}^{e x t}(t)=\int_{\partial \Omega_{e} \cap \partial_{N} \mathcal{B}} \overline{\mathbf{T}} N^{a} d A
\end{aligned}
$$

${ }_{685} \overline{\mathbf{T}}$ being the traction applied over the Newman boundary, and the index $A=$ $1, \ldots N$.

\section{Appendix B.1.2. Diffusion problem}

The strong form of the diffusion problem consists of solving Eq. (B.10) with Dirichlet Eq. (B.11) and Neumann boundary conditions. Eq. (B.12) and the initial condition Eq. (B.13).

$$
\begin{cases}\dot{c}+\operatorname{Div} \mathbf{J}=0 & \text { on } \partial_{D} \mathcal{B} \\ c=\bar{c} & \text { on } \partial_{N} \mathcal{B} \\ \mathbf{J} \cdot \mathbf{N}=\bar{J} & \text { at } t=0 \\ c=c_{0} & \end{cases}
$$

\footnotetext{
${ }^{18}$ in order to avoid confusion with other symbols, calligraphic notation is used within this section to indicate the vector of nodal forces and the stiffness and mass matrix.
} 
The diffusion problem discretized in space and time has become equivalent to the following equation in the unknowns $\Delta \mathbf{c}, \Delta \boldsymbol{\mu}^{\mathrm{M}}$

$$
\left(\frac{\mathcal{M}}{\Delta t}+\mathcal{K}^{c c}\right) \Delta \mathbf{c}+\mathcal{K}^{c \mu^{M}} \Delta \boldsymbol{\mu}^{\mathrm{M}}=\mathcal{R}^{c}
$$

In reference to the diffusion potential of Eq. (48), the stiffness matrix blocks $\mathcal{K}^{c c}$ and $\mathcal{K}^{c \mu^{M}}$ in Eq. (B.14) can be computed as

$$
\begin{aligned}
\mathcal{K}^{c c}= & \int_{\Omega_{e}}\left[M\left(1+\frac{c_{\max }}{c\left(c_{\max }-c\right)}+\frac{\partial \ln \gamma}{\partial \ln c}\right) \mathbf{C}^{-1} \nabla N^{b} \cdot \nabla N^{a}+\right. \\
& \left.\frac{M}{R T} N^{b} \mathbf{C}^{-1} \nabla \mu^{M} \cdot \nabla N^{a}+z_{i} F \frac{M}{R T} N^{b} \mathbf{C}^{-1} \nabla \phi \cdot \nabla N^{a}\right] d V \\
& \mathcal{K}^{c \mu^{M}}=\int_{\Omega_{e}} \frac{M}{R T} c \mathbf{C}^{-1} \nabla N^{b} \cdot \nabla N^{a} d V
\end{aligned}
$$

and the residual $\mathcal{R}^{c}$ has the following expression

$$
\mathcal{R}^{c}=\left.\mathcal{F}_{c}^{e x t}\right|_{n+1}-\left.\mathcal{F}_{c}^{\text {int }(k)}\right|_{n+1}-\frac{\mathcal{M}}{\Delta t}\left(\mathbf{c}_{n+1}^{(k)}-\mathbf{c}_{n}\right)
$$

690

Appendix B.1.3. Diffusion potential

From the diffusion potential of Eq. (48) we define the variable $\mu^{M}$ as the stress dependent component of the diffusion potential such that

$$
\mu^{M}+\frac{\beta}{\rho_{h}} \operatorname{Tr}\left(\mathbf{F}^{e T} \mathbf{P}\right)-\frac{\partial \Upsilon^{M}\left(\mathbf{C}^{e}, c_{i}\right)}{\partial c_{i}}=0
$$

The choice of $\mu^{M}$, together with displacement and concentration, as unknown variables will lead us to a mixed finite elements formulation.

To simplify the derivation we assume here $\partial \Upsilon^{M}\left(\mathbf{C}^{e}, c_{i}\right) / \partial c_{i}=0$.

The discrete form of Eq. B.18) reads

$$
\mathcal{M} \boldsymbol{\mu}^{\mathrm{M}}+\mathcal{F}_{\mu^{M}}^{\text {int }}=\mathbf{0}
$$

The internal forces vector has the following definition

$$
\mathcal{F}_{\mu^{M}}^{i n t}=\int_{\Omega_{e}} \frac{\beta}{\rho_{h}} \operatorname{Tr}\left(\mathbf{F}^{e T} \mathbf{P}\right) N^{a} d V
$$

The blocks of the stiffness matrix are computed according to

$$
\begin{gathered}
\mathcal{K}^{\mu^{M} u}=\int_{\Omega_{e}} \beta\left[\left(\mathbb{C}: \nabla N^{b}\right): \mathbf{F}^{e}+\mathbf{P}:\left(\mathbf{F}^{a-1} \nabla N^{b}\right)\right] N^{a} d V \\
\mathcal{K}^{\mu^{M} c}=\int_{\Omega_{e}} \frac{\beta}{\rho_{h}}\left[\frac{\partial \mathbf{P}}{\partial c}: \mathbf{F}^{e}\right] N^{a} N^{b} d V
\end{gathered}
$$


In summary, the electrochemical-mechanical problem to be solved at each iteration of the Newton-Raphson algorithm consists of the following non-symmetric system of three coupled equations in the unknowns $\mathbf{u}, \Delta \mathbf{c}, \Delta \boldsymbol{\mu}^{\mathbf{M}}$

$$
\left\{\begin{array}{l}
\mathcal{K}^{u u} \mathbf{u}+\mathcal{K}^{u c} \Delta \mathbf{c}=\mathcal{R}^{u} \\
\left(\frac{\mathcal{M}}{\Delta t}+\mathcal{K}^{c c}\right) \Delta \mathbf{c}+\mathcal{K}^{c \mu^{M}} \Delta \boldsymbol{\mu}^{\mathbf{M}}=\mathcal{R}^{c} \\
\mathcal{M} \Delta \boldsymbol{\mu}^{\mathbf{M}}+\mathcal{K}^{\mu^{M} u} \mathbf{u}+\mathcal{K}^{\mu^{M} c} \Delta \mathbf{c}=\mathcal{R}^{\mu^{M}}
\end{array}\right.
$$

The model has been implemented in an in-house object oriented $\mathrm{C}++$ numerical code. Grid handling and discretization make use of the deal.II finite-element library, an open-source library widely used in scientific computing [41] 39. Furthermore, it employs parallel processing, i.e. the simultaneous use of more than one CPU to execute the most time consuming calculations, i.e assembly of stiffness matrix and residual, and the solution of the linear system at each Newton iteration.

Appendix B.2. Constitutive Laws

As a quick reference for the reader, we recall here the main constitutive laws (with a reference to the corresponding equation in the article) adopted for the applications in Sections 4.1.1, 4.2, and 4.3.

- Multiplicative decomposition of the total deformation gradient, with anelastic isotropic volumetric expansion of the form Eq.17)

$$
\mathbf{F}=\mathbf{F}^{e} \mathbf{F}^{a} \quad \mathbf{F}^{a}\left(c_{i}\right)=\left(1+\frac{1}{\rho_{h}} \sum_{i} \beta_{i} c_{i}\right) \mathbf{I}
$$

- The finite element formulation for an electro-chemo-mechanical system is based on a free energy with two components as in Eq. 29p

$$
\Upsilon=\Upsilon^{M}\left(\mathbf{C}^{e}, c_{i}\right)+\Upsilon^{E C}\left(c_{i}, \phi\right)
$$

The function $\Upsilon^{M}$ determines the elastic response of the material (Eq. 441)

$$
\Upsilon^{M}\left(I_{1}\left(\mathbf{C}^{e}\right), J\left(\mathbf{C}^{e}\right), c_{i}\right)=\frac{1}{2} \lambda(\log J)^{2}-G \log J+\frac{1}{2} G\left(I_{1}-3\right)
$$

$\Upsilon^{E C}$ represents the electrochemical free energy $(\sqrt{47})$ ). In the case of diffusing neutral species it takes the form in Eq. (35)

$$
\Upsilon^{E C}\left(c_{i}, c_{V_{i}}\right)=c_{i} R T \ln c_{i}+c_{V_{i}} R T \ln c_{V_{i}}+\Upsilon_{I I}^{E C}\left(c_{i}, c_{V_{i}}\right)
$$

To study the transport of ions we adopted, instead, the free energy density given Eq. 477)

$$
\Upsilon^{E C}\left(c_{i}, c_{V_{i}}\right)=c_{i} R T \ln c_{i}+c_{V_{i}} R T \ln c_{V_{i}}+\Upsilon_{I I}^{E C}\left(c_{i}, c_{V_{i}}\right)+z_{i} F c_{i} \phi
$$


- The diffusion potential for the charged species $i$, in presence of a network constraint on the number of $i$-sites available and including stress effects, is stated in Eq. 48 as

$$
\begin{aligned}
& \varpi_{i}\left(\mathbf{C}^{e}, c_{i}\right)=\frac{\partial \Upsilon^{M}\left(\mathbf{C}^{e}, c_{i}\right)}{\partial c_{i}}+\frac{\partial \Upsilon^{E C}\left(c_{i}, \phi\right)}{\partial c_{i}}-\frac{\beta_{i}}{\rho_{h}} \operatorname{Tr}\left(\mathbf{F}^{e T} \mathbf{P}\right)= \\
& =\frac{\partial \Upsilon^{M}\left(\mathbf{C}^{e}, c_{i}\right)}{\partial c_{i}}+R T \ln \left(\frac{c_{i}}{c_{i_{\max }}-c_{i}}\right)+R T \ln \bar{\gamma}_{i}+z_{i} F \phi-\frac{\beta_{i}}{\rho_{h}} \operatorname{Tr}\left(\mathbf{F}^{e T} \mathbf{P}\right)
\end{aligned}
$$

with the term

$$
\frac{\partial \Upsilon^{M}\left(\mathbf{C}^{e}, c_{i}\right)}{\partial c_{i}}=\frac{1}{2} \lambda_{1}(\log J)^{2}-G_{1} \log J+\frac{1}{2} G_{1}\left(I_{1}-3\right)
$$

expressing the dependence of the elastic properties on concentration.

- The first Piola-Kirchhoff stress derived from Eq. (41) according to Eq. 24. is stated in Eq. 43) as

$$
\mathbf{P}=\frac{\partial \Upsilon^{M}}{\partial \mathbf{F}}=\frac{\partial \Upsilon^{M}}{\partial \mathbf{F}^{e}} \frac{\partial \mathbf{F}^{e}}{\partial \mathbf{F}}=\left[(\lambda \log J-G) \mathbf{F}^{e-T}+G \mathbf{F}^{e}\right] \mathbf{F}^{a-T}
$$

\section{References}

[1] F. Larché, J. W. Cahn, The interaction of composition and stress in crystalline solids., Acta Metallurgica 33 (1985) 331-367.

[2] C. Truesdell, W. Noll, The Nonlinear Field Theories of Mechanics, Springer, Berlin, 1965.

[3] M. Planck, A linear theory of thermochemical equilibrium of solids under stress., Annalen der Physik (Leipzig) 30 (1887) 563.

[4] E. A. Guggenheim, Thermodynamics, North-Holland Publishing Company, Amsterdam, 1967.

[5] C. Truesdell, Rational Thermodynamics, Springer, New York, 1969.

[6] Q. Yang, L. Stainier, M. Ortiz, A variational formulation of the coupled thermo-mechanical boundary-value problem for general dissipative solids., Journal of the Mechanics and Physics of Solids 54 (2005) 401-424.

[7] A. F. Bower, P. R. Guduru, S. V. A., A finite strain model of stress, diffusion, plastic flow, and elecrochemical reactions in lithium-ion half-cell., Journal of the Mechanics and Physics of Solids 59 (2011) 804-828.

[8] A. F. Bower, P. R. Guduru, A simple finite element model of diffusion, finite deformation, plasticity and fracture in lithium ion insertion electrode materials, Modelling and Simulation in Materials Science and Engineering 20 (4) (2012) 045004. 
[9] L. Anand, A cahn-hilliard-type theory for species diffusion coupled with large elastic-plastic deformations., Journal of the Mechanics and Physics of Solids 60 (2012) 1983-2002.

[10] K. Zhao, M. Pharr, S. Cai, J. J. Vlassak, Z. Suo, Large plastic deformation in high-capacity lithium-ion batteries caused by charge and discharge., Journal of the American Ceramic Society 94 (2011) S226-S235.

[11] C. H. Wu, The role of eshelby stress in composition-generated and stressassisted diffusion., Journal of the Mechanics and Physics of Solids 40 (2001) 1771-1794. doi:http://dx.doi.org/10.1016.

[12] R. A. Toupin, The elastic dielectic., Journal of Rational Mechanics and Analysis 5 (1956) 849-915.

[13] R. W. Ogden, D. J. Steigmann, Mechanics and electrodynamics of magnetoand electro-elastic materials., CISM courses and lectures: no. 527, Wien ; New York : Springer, c2011., 2011.

[14] A. J. Bard, G. Inzelt, F. Scholz, Electrochemical Dictionary, Springer, Berlin, 2012.

[15] W. Lee, J. W. Han, Y. Chen, Z. Cai, B. Yildiz, Cation size mismatch and charge interactions drive dopant segregation at the surfaces of manganite perovskites, Journal of the American Chemical Society 135 (2013) 79097925. doi:10.1021/ja3125349.

[16] W. Jung, H. L. Tuller, Investigation of surface sr segregation in model thin film solid oxide fuel cell perovskite electrodes, Energy \& Environmental Science 5 (2012) 5370-5378. doi:10.1039/C1EE02762J.

[17] H. Jalili, J. W. Han, Y. Kuru, Z. Cai, B. Yildiz, New insights into the strain coupling to surface chemistry, electronic structure, and reactivity of $\mathrm{La}_{0.7} \mathrm{Sr}_{0.3} \mathrm{MnO}_{3}$, Journal of Physical Chemistry Letters 2 (7) (2011) 801807. doi:10.1021/jz200160b.

[18] T. T. Fister, D. D. Fong, J. A. Eastman, P. M. Baldo, M. J. Highland, P. H. Fuoss, K. R. Balasubramaniam, J. C. Meador, P. A. Salvador, In situ characterization of strontium surface segregation in epitaxial $\mathrm{La}_{0.7} \mathrm{Sr}_{0.3} \mathrm{MnO}_{3}$ thin films as a function of oxygen partial pressure, Applied Physics Letters 93 (15) (2008) -. doi:http://dx.doi.org/10.1063/1.2987731.

[19] H. Dulli, P. A. Dowben, S.-H. Liou, E. W. Plummer, Surface segregation and restructuring of colossal-magnetoresistant manganese perovskites

口 $\quad \mathrm{la}_{0.65} \mathrm{sr}_{0.35} \mathrm{mno}_{3}$, Phys. Rev. B 62 (2000) R14629-R14632. doi:10.1103/ PhysRevB.62.R14629

[20] M. Martin, Oxygen Potential Gradient Induced Degradation of Oxides, 765 J Journal of the Korean Ceramic Society 49 (1) (2012) 29-36. doi:10.4191/ kcers.2012.49.1.029. 
[21] D. Neagu, G. Tsekouras, D. N. Miller, H. Ménard, J. T. S. Irvine, In situ growth of nanoparticles through control of non-stoichiometry., Nature chemistry 5 (October) (2013) 916-23. doi:10.1038/nchem.1773.

[35] J. Newman, T.-A. K. E., Electrochemical Systems, John Wiley \& Sons, Inc., Hoboken, New Jersey, 2004. 
[36] F. A. Kröger, F. J. Vink, Relations between the concentrations of imperfections in crystalline solids., Solid State Physics 3 (1956) 307-435.

[37] Y. Gao, M. Zhou, Coupled mechano-diffusional driving forces for fracture in electrode materials, Journal of Power Sources 230 (2013) 176 - 193. doi:http://dx.doi.org/10.1016/j.jpowsour.2012.12.034.

[38] G. Bucci, S. P. Nadimpalli, V. A. Sethuraman, A. F. Bower, P. R. Guduru, Measurement and modeling of the mechanical and electrochemical response of amorphous si thin film electrodes during cyclic lithiation, Journal of the Mechanics and Physics of Solids 62 (2014) 276 - 294, sixtieth anniversary issue in honor of Professor Rodney Hill. doi:http://dx.doi.org/10. $1016 / j \cdot j m p s .2013 .10 .005$.

[39] W. Bangerth, T. Heister, L. Heltai, G. Kanschat, M. Kronbichler, M. Maier, B. Turcksin, T. D. Young, The deal.II library, version 8.2, Archive of Numerical Software 3.

[40] S. A. Chester, C. V. D. Leo, L. Anand, A finite element implementation of a coupled diffusion-deformation theory for elastomeric gels, International 1. Journal of Solids and Structures 52 (2015) 1 - 18. doi:http://dx.doi. org/10.1016/j.ijsolstr.2014.08.015.

[41] W. Bangerth, R. Hartmann, G. Kanschat, deal.II - a general purpose object oriented finite element library, ACM Trans. Math. Softw. 33 (4) (2007) $24 / 1-24 / 27$. 
Graphical Abstract

\section{Evolution of Ambipolar Species Concentration in the Presence of Stress}
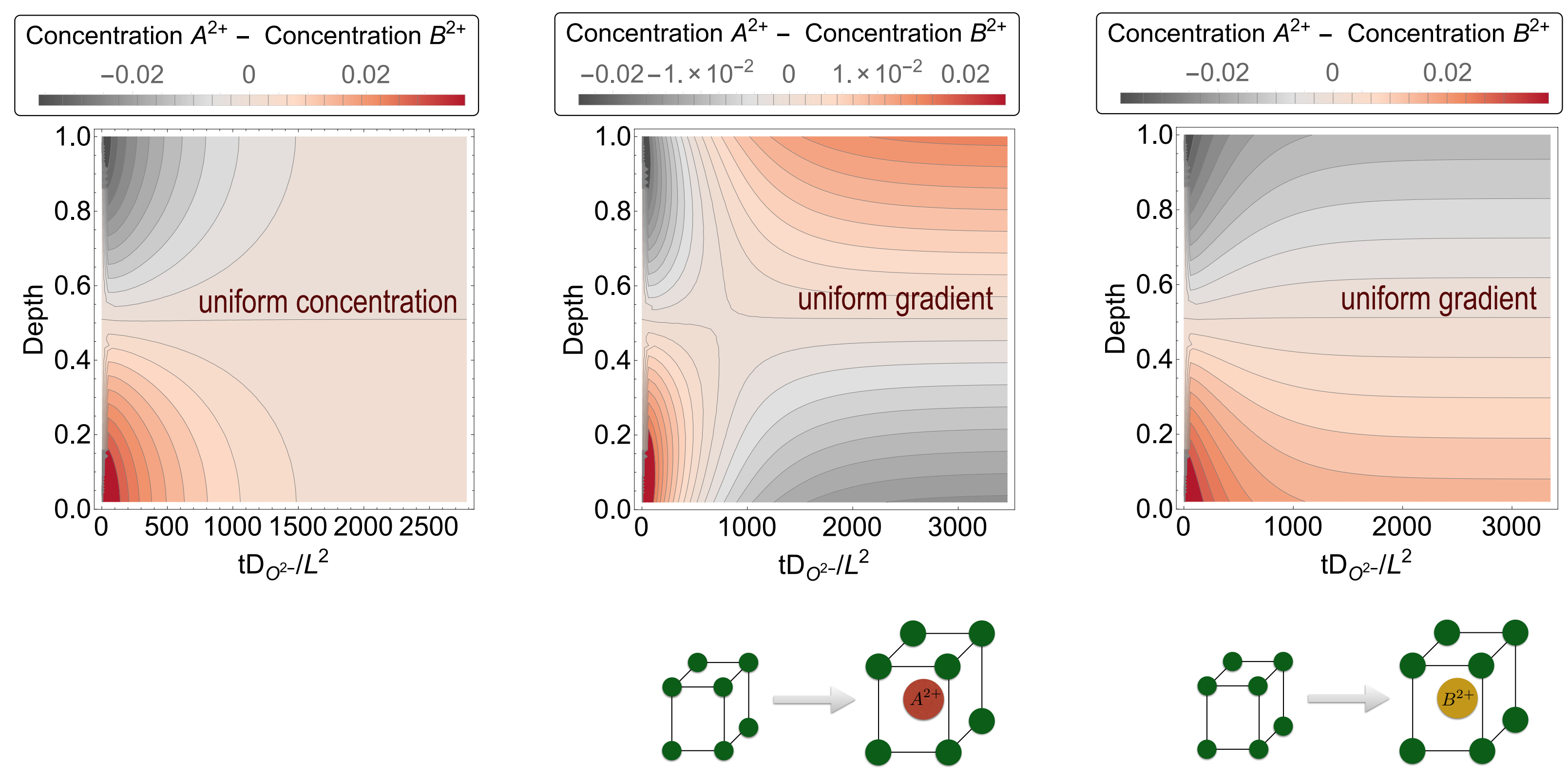